\title{
Sensitivity of quantum walks to a boundary of two-dimensional lattices: approaches based on the CGMV method and topological phases
}

\author{
Takako Endo, ${ }^{1}$ Norio Konno, ${ }^{2}$ Hideaki Obuse, ${ }^{3}$ Etsuo Segawa. ${ }^{4} *$ \\ ${ }^{1}$ Institute for Global Leadership, Ochanomizu University \\ 2-1-1 Ohtsuka, Bunkyo, Tokyo, 112-0012, Japan \\ ${ }^{2}$ Department of Applied Mathematics, Faculty of Engineering, Yokohama National University \\ Hodogaya, Yokohama 240-8501, Japan \\ ${ }^{3}$ Department of Applied Physics, Hokkaido University \\ Sapporo, Hokkaido 060-8628, Japan \\ ${ }^{4}$ Graduate School of Information Sciences, Tohoku University, \\ Aoba, Sendai 980-8579, Japan
}

\begin{abstract}
In this paper, we treat quantum walks in a two-dimensional lattice with cutting edges along a straight boundary introduced by Asboth and Edge [Phys. Rev. A 91, 022324 (2015)] in order to study one-dimensional edge states originating from topological phases of matter and to obtain collateral evidence of how a quantum walker reacts to the boundary. Firstly, we connect this model to the CMV matrix, which provides a 5-term recursion relation of the Laurent polynomial associated with spectral measure on the unit circle. Secondly, we explicitly derive the spectra of bulk and edge states of the quantum walk with the boundary using spectral analysis of the CMV matrix. Thirdly, while topological numbers of the model studied so far are well-defined only when gaps in the bulk spectrum exist, we find a new topological number defined only when there are no gaps in the bulk spectrum. We confirm that the existence of the spectrum for edge states derived from the CMV matrix is consistent with the prediction from a bulk-edge correspondence using topological numbers calculated in the cases where gaps in the bulk spectrum do or do not exist. Finally, we show how the edge states contribute to the asymptotic behavior of the quantum walk through limit theorems of the finding probability. Conversely, we also propose a differential equation using this limit distribution whose solution is the underlying edge state. ॥
\end{abstract}

\section{Introduction}

Quantum walks are the quantum analog of random walks, first introduced in [17]. The initial impetus for intensively studying quantum walks came from the field of quantum

*e-segawa@m.tohoku.ac.jp

Key words and phrases. quantum walks, topological phase, CMV matrix 
information [1]. Nowadays, overlaps between quantum walks and quantum information, as well as, various other research fields have been found and established by interdisciplinary researches (see [26, 30] and references therein).

One recent area of studying is connecting localization in quantum walks with topological phases of matter, i.e., topological insulators, which is currently a hot field in condensedmatter physics. Kitagawa et al. [20, 22] introduced a quantum walk as a model for exploring the topological phases of matter, which is simplified by discretizing temporal and spatial spaces. In particular, quantum walks on a one-dimensional lattice, whose unitary time-evolution operator is spatially inhomogeneous and retains chiral symmetry, have been intensively studied (e.g., [2, 10, 11, 13, 14, 29, 28]). Kitagawa[22] also found a connection between two-dimensional topological insulators and quantum walk models on a two-dimensional square lattice $(x, y) \in \mathbb{Z}^{2}$ [15, 16]. Asboth and Edge further studied on this model with a "cutting edge" [4], meaning that all the edges connecting vertices at $(0, y) \in \mathbb{Z}^{2}$ and $(-1, y) \in \mathbb{Z}^{2}$ for $y \in \mathbb{Z}$ are cut and rewired to make self-loops on $(0, y)$ and $(-1, y)$. This model is determined by a pair of parameters $(\alpha, \beta) \in[0,2 \pi)^{2}$; the first and second parameters, $\alpha$ and $\beta$, determine the local dynamics of the horizontal and vertical directions on the two-dimensional lattice, respectively. The local dynamics are provided by alternatively allowing the two-dimensional rotation matrices with angles of $\alpha$ and $\beta$ to act up on twodimensional internal states; we call this the AE model. See for a detailed definition of this graph setting and the time evolution in Section 2. Asboth and Edge provided analytical and numerical results showing the existence of unidirectional edge states along the self-loops.

Due to the unitarity of the time evolution of quantum walks, we obtain a sequence of probability distributions for each time step $\left\{\rho_{n}\right\}_{n \in \mathbb{N}}$. Let $\psi_{n}$ be a quantum state at time $n$ that is obtained by the $n$-th iteration of the unitary time evolution to an initial state. We call the map $\psi_{n} \mapsto \rho_{n}$ a "measurement" which is represented by an orthogonal projection map, see Section 2.2 (3) for more detail. In this paper, we provide further analytical results on the AE model connecting this measurement. The main purpose of this paper is to determine whether it is possible to estimate spectral information about the AE model by obtaining the distribution, $\rho_{n}$, after measuring the quantum state $\psi_{n}$, and if so, which of the spectral properties are reflected to the limit behavior of $\rho_{n}$.

To this end, we first connect the AE model to the CMV matrix (Theorem 1). The CMV matrix represents the five-term recursion relation for the orthogonal Laurent polynomials associated with a given positive measure on the unit circle in the complex plane [7]. The authors Cantero, Grünbaum, Moral, and Velázquez first connected the CMV matrix to quantum walks in [9]. We call spectral analysis of quantum walks using this connection to the CMV matrix the CGMV method after these authors' initials [25]. This method enable us to obtain spectral information about our model in quite-explicit form. Due to the translation symmetry with respect to the direction parallel to the boundary of the cutting edges of the AE model, we can take the Fourier transform and decompose the time operator $U^{2}$ restricted to the subspace generated by horizontal arcs into the unitary operators $\left\{\hat{\Gamma}_{k}\right\}_{k \in[0,2 \pi)}$. Interestingly, we find that $\hat{\Gamma}_{k}$ for each $k$ is unitarily equivalent to the CMV matrix with null odd Verblunsky parameters

$$
(\eta(k), 0, \eta(k), 0, \ldots), \quad \eta(k)=\sin (\alpha-\beta) \cos k+i \sin (\alpha+\beta) \sin k .
$$

Thanks to this connection, secondly, we can derive the spectra for the bulk and edge states. 
We obtain the bulk spectrum, in which gaps may exist and the spectrum for the edge state, which may appear in the gaps of the bulk spectrum (Theorem 2). As a corollary, we can classify the spectra according to the signs given by

$$
(\operatorname{sgn}(\sin 2 \alpha \sin 2 \beta), \operatorname{sgn}(\sin (\alpha+\beta)), \operatorname{sgn}(\sin (\alpha-\beta))) .
$$

Thirdly, in contrast to the topological number in Ref. [4] which is defined only when gaps in the bulk spectrum exist, we derive a new topological number which is defined only when there are no gaps in the bulk spectrum. We determine the relationship between the edge spectrum derived from Theorem 2 and topological numbers calculated in the cases where gaps in the bulk spectrum do or do not exist in figures $2-5$.

Fourthly, we examine how the boundary affects the quantum walk in the two-dimensional case (Theorem 3). On the one-dimensional lattice, if the edge state exists, then we observe localization (i.e., the time-averaged limit measure is strictly positive) as the asymptotic behavior of the sequence of the probability distributions $\left\{\rho_{n}\right\}_{n}$ [13, 29]. To express the response of the quantum walk to the boundary in the two-dimensional case, we define $\nu_{n}$ : $\partial V \rightarrow[0,1]$ such that $\nu_{n}(j)$ is the finding probability of the AE model at the $n$-th iteration and at self-loops on $[0, j] \in \mathbb{Z}_{+} \times \mathbb{Z}$ starting from the self-loop at the origin. We show that the response is classified into four behaviors depending on the values of the parameters $(\alpha, \beta)$ :

(1) continuous linear spreading;

(2) ballistic spreading;

(3) localization;

(4) null.

In particular, the limit-density function of (10) is described using the Konno distribution [23, 24]. The limit behavior of the quantum walk contributed by the edge state in the case of (2) is completely expressed in Theorem 4.

As a consequence, by assuming the group velocity $v(k)$, quasi-effective mass $M(k)$, and density of the edge state $m_{0}(k)$, we obtain a parametric plot of the limit-density function of (2) using these physical quantities (see Corollary 3.) By this parametric plot expression, conversely, we can estimate the underlying edge state from the obtained distribution. More precisely, if we have an estimate $\tilde{g}$ of the true limit distribution $g$ from the distribution data obtained by measurement of self-loops after finite unitary time iterations, then the underlying edge state can be also estimated from this data by solving a differential equation with some constant $c \in \mathbb{R} ; \tilde{g}(v) d v / d k=c v^{2}$, implying that the group velocity of edge state is an inverse of Konno distribution in this model (see Corollary 4). We note that comparing discrete-time quantum walks with the discrete-Schrödinger operator on lattices [5, 18, 34] is one of interesting problems, which should be addressed in future.

This paper is organized as follows. In Sect. 2, we define the graph and the quantum walk treated here. In Sect. 3, we connect the quantum walk with the CMV matrix. Section 4 is devoted to the spectral analysis and topological phases of the AE model. In Sect. 5, we present how the properties of the spectrum obtained in the previous section are reflected in the stochastic behavior along the boundary by studying limit distribution functions. Finally, in Sect. 6, we present limit distribution functions toward the bulk, which clearly exhibit exponential decay. 


\section{Model}

(a)

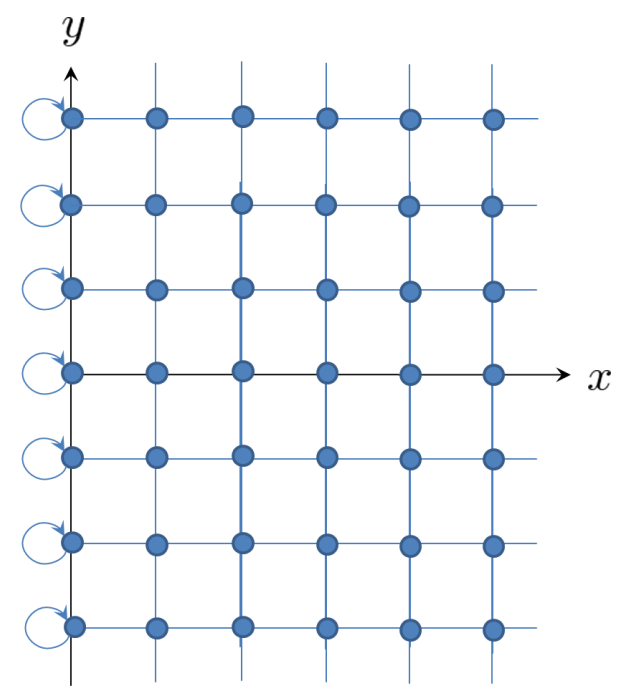

(b)
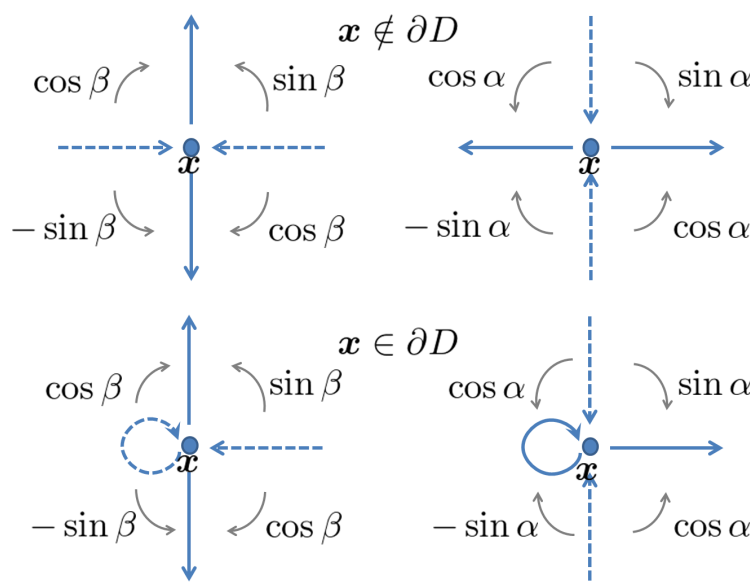

Figure 1: (a) The graph treated in the present work. (b) The one-step time evolution $U$ at a vertex $\boldsymbol{x} \notin \partial V$ and $\boldsymbol{x} \in \partial V$. The dotted and solid arcs represent the input and output arcs, respectively.

\subsection{Graph}

Let $G=(V, A)$ be a directed graph whose vertex set and arc set are $V$ and $A$, respectively. In this paper, we express the vertex set as

$$
V=\mathbb{Z}_{+} \times \mathbb{Z}=\left\{(x, y) \mid x \in \mathbb{Z}_{+}, y \in \mathbb{Z}\right\}
$$

where $\mathbb{Z}$ is the integer set and $\mathbb{Z}_{+}$is the non-negative integer set. We denote the boundary of this graph by $\partial V:=\{(0, j) \in V \mid j \in \mathbb{Z}\}$. The arc set $A$ is represented by

$$
A=\{(\boldsymbol{x} ; d) \mid \boldsymbol{x} \in V, d \in\{0,1,2,3\}\} .
$$

For $a \in A$, the origin and terminus of $a$ are denoted by $o(a), t(a) \in V$, respectively, and the inverse of $a$ is denoted by $\bar{a}$. Here if $a=(\boldsymbol{x} ; d) \in A$, then

$$
\begin{aligned}
& t(a)=\boldsymbol{x}, \\
& o(a)= \begin{cases}\boldsymbol{x}+(1,0) & : d=0, \\
\boldsymbol{x} & : d=1, \boldsymbol{x} \in \partial V, \\
\boldsymbol{x}-(1,0) & : d=1, \boldsymbol{x} \notin \partial V, \\
\boldsymbol{x}+(0,1) & : d=2, \\
\boldsymbol{x}-(0,1) & : d=3,\end{cases}
\end{aligned}
$$


which means that $(\boldsymbol{x} ; d)$ is the arc coming from the direction labeled by $d$ whose terminus is $\boldsymbol{x}$; see figure 1. We call $\{(\boldsymbol{x} ; 1) \mid \boldsymbol{x} \in \partial V\} \subset A$ a set of self-loops. We take $\bar{a}=a$ for every self-loop.

\subsection{Definition of the AE model}

The one-step time evolution of the AE model is explained as follows: the incident state coming from the horizontal (vertical) direction is transmitted at the terminal vertex to the vertical (horizontal) directions with some complex-valued weight conserving the unitarity. Here, we define the model in the arc-set representation $\ell^{2}(A)$ first and then, convert the representation to the vertex-set ones; $\ell^{2}\left(V ; \mathbb{C}^{4}\right)$ or $\ell^{2}\left(V ; \mathbb{C}^{2}\right)$.

(1) Total Hilbert space: $\mathcal{A}:=\ell^{2}(A)=\{\psi: A \rightarrow \mathbb{C} \mid\|\psi\|<\infty\}$. The inner product is the standard inner product. Now we introduce a binary relation of $A$ :

$$
a \stackrel{\pi}{\sim} b \Leftrightarrow t(a)=t(b)
$$

Since $\pi$ is an equivalence relation, we obtain the quotient sets by

$$
A / \pi=\oplus_{\boldsymbol{x} \in V} A_{\boldsymbol{x}}
$$

where $A_{x}=\{a \in A \mid t(a)=x\}$. From this partition of $A$, we set the orthogonal decomposition of $\mathcal{A}$ into

$$
\mathcal{A}=\bigoplus_{x \in V} \mathcal{A}_{x}
$$

Here

$$
\mathcal{A}_{\boldsymbol{x}}=\left\{\psi \in \mathcal{A} \mid a \notin A_{\boldsymbol{x}} \Rightarrow \psi(a)=0\right\} .
$$

(2) Time evolution: We assign the local unitary operator on $\mathcal{A}_{\boldsymbol{x}}$ for each $\boldsymbol{x} \in V$; this operator acts alternatively in the horizontal and vertical directions in the following way

$$
\begin{aligned}
& C_{\boldsymbol{x}} \delta_{(\boldsymbol{x} ; 0)}=\cos \beta \delta_{(\boldsymbol{x} ; 3)}+\sin \beta \delta_{(\boldsymbol{x} ; 2)}, \\
& C_{\boldsymbol{x}} \delta_{(\boldsymbol{x} ; 1)}=-\sin \beta \delta_{(\boldsymbol{x} ; 3)}+\cos \beta \delta_{(\boldsymbol{x} ; 2)}, \\
& C_{\boldsymbol{x}} \delta_{(\boldsymbol{x} ; 2)}=\cos \alpha \delta_{(\boldsymbol{x} ; 1)}+\sin \alpha \delta_{(\boldsymbol{x} ; 0)}, \\
& C_{\boldsymbol{x}} \delta_{(\boldsymbol{x} ; 3)}=-\sin \alpha \delta_{(\boldsymbol{x} ; 1)}+\cos \alpha \delta_{((\boldsymbol{x} ; 0)},
\end{aligned}
$$

where $\delta_{a} \in \mathcal{A}$ is the Kronecker delta function, that is,

$$
\delta_{a}\left(a^{\prime}\right)= \begin{cases}1 & : a=a^{\prime}, \\ 0 & : a \neq a^{\prime} .\end{cases}
$$

The pair of parameters, $(\alpha, \beta) \in[0,2 \pi)^{2}$, which determines this quantum walk, will be important for providing its behavior. The time evolution is denoted by the iteration of the unitary map, $U: \mathcal{A} \rightarrow \mathcal{A}$

$$
(U \psi)(a)=\sum_{b: t(b)=o(a)}\left\langle\delta_{\bar{a}}, C_{o(a)} \delta_{b}\right\rangle \psi(b) .
$$

See also figure 1. 
(3) Distribution: Throughout this paper, we fix the initial state $\psi_{0} \in \mathcal{A}$ by $\psi_{0}(a)=\delta_{(\mathbf{0} ; 1)}(a)$ which represents the self-loop at the origin. We define the distribution at each time step $n, \rho_{n}: A \rightarrow[0,1]$

$$
\rho_{n}(a)=\left|\psi_{n}(a)\right|^{2}=\left\|\Pi_{\delta_{a}} \psi_{n}\right\|^{2},
$$

where for a subset $\mathcal{A}^{\prime} \subset \mathcal{A}, \Pi_{\mathcal{A}^{\prime}}$ is the projection operator onto $\mathcal{A}^{\prime}$. Here $\psi_{n}=U^{2 n} \psi_{0}$. Obviously $\sum_{a \in A} \Pi_{\delta_{a}}=1$ holds. We regard $\rho_{n}(a)$ as the finding probability of the quantum walk at position $a \in A$ and at time $2 n$. We observe how this quantum walk recognizes the boundary of the graph through the asymptotic behavior of $\nu_{n}(j):=$ $\rho_{n}((0, j) ; 1)$ for large $n$.

Remark 1. The unitarity of the time evolution $U$ is immediately shown by the product of the two unitary operators of $U ; U=S C$, where

$$
S \psi(a)=\psi(\bar{a}), \quad C=\oplus \sum_{\boldsymbol{x} \in V} C_{\boldsymbol{x}}
$$

The unitary operators $S$ and $C$ are called shift and coin operators, respectively.

\subsection{An alternative quantum walk}

The horizontal and vertical arcs are denoted by

$$
A^{(\leftrightarrow)}=\{(\boldsymbol{x} ; d) \in A \mid d \in\{0,1\}\}, \quad A^{(\uparrow)}=\{(\boldsymbol{x} ; d) \in A \mid d \in\{2,3\}\},
$$

respectively. We set the associated subspaces

$$
\mathcal{A}^{(\leftrightarrow)}=\left\{\psi \in \mathcal{A} \mid a \notin A^{(\leftrightarrow)} \Rightarrow \psi(a)=0\right\}, \quad \mathcal{A}^{(\uparrow)}=\left\{\psi \in \mathcal{A} \mid a \notin A^{(\uparrow)} \Rightarrow \psi(a)=0\right\} .
$$

We define unitary maps $\mathcal{U}$ and $\mathcal{U}_{e}$ which convert the Hilbert space in the arc-set representations to ones in the vertex set representations with four and two internal states, respectively. These maps are given by $\mathcal{U}: \mathcal{A} \rightarrow \ell^{2}\left(V ; \mathbb{C}^{4}\right)$ and $\mathcal{U}_{e}: \mathcal{A}^{(\leftrightarrow)} \rightarrow \ell^{2}\left(V ; \mathbb{C}^{2}\right)$, where

$$
\ell^{2}\left(V ; \mathbb{C}^{n}\right)=\left\{f: V \rightarrow \mathbb{C}^{n} \mid \sum_{x \in V}\|f(\boldsymbol{x})\|_{\mathbb{C}^{n}}^{2}<\infty\right\} \quad(n \in\{1,2, \ldots\}) .
$$

More precisely, these maps $\mathcal{U}$ and $\mathcal{U}_{e}$ are defined as follows.

\section{Definition 1.}

We define $\mathcal{U}: \mathcal{A} \rightarrow \ell^{2}\left(V ; \mathbb{C}^{4}\right)$ such that

$$
(\mathcal{U} \psi)(\boldsymbol{x})={ }^{T}[\psi(\boldsymbol{x} ; 0) \quad \psi(\boldsymbol{x} ; 1) \quad \psi(\boldsymbol{x} ; 2) \quad \psi(\boldsymbol{x} ; 3)] .
$$

We also define $\mathcal{U}_{e}: \mathcal{A}^{(\leftrightarrow)} \rightarrow \ell^{2}\left(V ; \mathbb{C}^{2}\right)$ such that

$$
\left(\mathcal{U}_{e} \psi\right)(\boldsymbol{x})={ }^{T}[\psi(\boldsymbol{x} ; 0) \quad \psi(\boldsymbol{x} ; 1)] .
$$


We can see that these inverse maps $\mathcal{U}^{-1}: \ell^{2}\left(V ; \mathbb{C}^{4}\right) \rightarrow \mathcal{A}$ and $\mathcal{U}_{e}^{-1}: \ell^{2}\left(V ; \mathbb{C}^{2}\right) \rightarrow \mathcal{A}^{(\leftrightarrow)}$ are expressed by

$$
\begin{aligned}
& \left(\mathcal{U}^{-1} f\right)(\boldsymbol{x} ; j)=f_{j}(\boldsymbol{x}) \quad(j \in\{0,1,2,3\}), \\
& \left(\mathcal{U}_{e}^{-1} g\right)(\boldsymbol{x} ; j)=g_{j}(\boldsymbol{x}) \quad(j \in\{0,1\})
\end{aligned}
$$

for $f \in \ell^{2}\left(V ; \mathbb{C}^{4}\right)$ with $f(\boldsymbol{y})={ }^{T}\left[f_{0}(\boldsymbol{y}) f_{1}(\boldsymbol{y}) f_{2}(\boldsymbol{y}) f_{3}(\boldsymbol{y})\right] \in \mathbb{C}^{4}$ and $g \in \ell^{2}\left(V ; \mathbb{C}^{2}\right)$ with $g(\boldsymbol{y})={ }^{T}\left[g_{0}(\boldsymbol{y}) g_{1}(\boldsymbol{y})\right] \in \mathbb{C}^{2}$. We put $|0\rangle={ }^{T}[1,0]$ and $|1\rangle={ }^{T}[0,1]$ as the standard basis of $\mathbb{C}^{2}$. Set the two-dimensional $\gamma$-rotation matrix by

$$
H_{\gamma}=\left[\begin{array}{cc}
\cos \gamma & -\sin \gamma \\
\sin \gamma & \cos \gamma
\end{array}\right] \cdot(\gamma \in[0,2 \pi))
$$

We define

$$
P_{\gamma}=|0\rangle\left\langle 0\left|H_{\gamma}, Q_{\gamma}=\right| 1\right\rangle\left\langle 1\left|H_{\gamma}, S_{\gamma}=\right| 1\right\rangle\langle 0| H_{\gamma} .
$$

For $\psi \in \ell^{2}\left(V ; \mathbb{C}^{4}\right)$ with $\psi(\boldsymbol{x})={ }^{T}\left[\psi_{0}(\boldsymbol{x}) \psi_{1}(\boldsymbol{x}) \psi_{2}(\boldsymbol{x}) \psi_{3}(\boldsymbol{x})\right] \in \mathbb{C}^{4}$, we denote $\psi^{(\leftrightarrow)}(\boldsymbol{x})=$

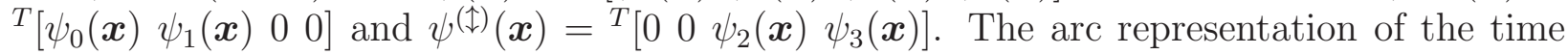
evolution of the quantum walk whose Hilbert space was $\ell^{2}(A)$ is converted to the vertex representation whose Hilbert space is $\ell^{2}\left(V ; \mathbb{C}^{4}\right)$ :

Lemma 1. Denote $U^{\prime}=\mathcal{U U U}^{-1}$. Then we have

$$
\begin{aligned}
& \left(U^{\prime} \psi^{(\leftrightarrow)}\right)(x, y)=\tilde{Q}_{\beta} \psi^{(\leftrightarrow)}(x, y-1)+\tilde{P}_{\beta} \psi^{(\leftrightarrow)}(x, y+1) \\
& \left(U^{\prime} \psi^{(\uparrow)}\right)(x, y)= \begin{cases}\tilde{Q}_{\alpha} \psi^{(\uparrow)}(x-1, y)+\tilde{P}_{\alpha} \psi^{(\uparrow)}(x+1, y) & :(x, y) \notin \partial V, \\
\tilde{S}_{\alpha} \psi^{(\uparrow)}(x, y)+\tilde{P}_{\alpha} \psi^{(\uparrow)}(x+1, y) & :(x, y) \in \partial V .\end{cases}
\end{aligned}
$$

Here

$\tilde{P}_{\alpha}:=|0\rangle\left\langle 1\left|\otimes P_{\alpha}, \tilde{Q}_{\alpha}:=\right| 0\right\rangle\left\langle 1\left|\otimes Q_{\alpha}, \tilde{P}_{\beta}:=\right| 1\right\rangle\left\langle 0\left|\otimes P_{\beta}, \tilde{Q}_{\beta}:=\right| 1\right\rangle\left\langle 0\left|\otimes Q_{\beta}, \tilde{S}_{\alpha}:=\right| 0\right\rangle\langle 1| \otimes S_{\alpha}$.

Proof. See Appendix A.

Note that if $\gamma=\gamma^{\prime}$, then $\tilde{X}_{\gamma} \tilde{X}_{\gamma^{\prime}}^{\prime}=0\left(X, X^{\prime} \in\{P, Q\}\right)$. This property reflects the quantum walker's alternation between moving in the vertical and horizontal directions. Therefore, we can easily observe that

$$
U^{2}\left(\mathcal{A}^{(\leftrightarrow)}\right) \subset \mathcal{A}^{(\leftrightarrow)}, \quad U^{2}\left(\mathcal{A}^{(\uparrow)}\right) \subset \mathcal{A}^{(\uparrow)} .
$$

Since our initial condition is $\psi_{0}=\delta_{(0 ; 1)} \in \mathcal{A}^{(\leftrightarrow)}$ which is the self-loop at the origin, we concentrate on $\mathcal{A}^{(\leftrightarrow)}$. Using Lemma 1, we obtain Lemma 2, see the detailed proof in Appendix B,

Lemma 2. Let $\Gamma: \ell^{2}\left(V ; \mathbb{C}^{2}\right) \rightarrow \ell^{2}\left(V ; \mathbb{C}^{2}\right)$ be a map defined by for $\varphi \in \ell^{2}\left(V ; \mathbb{C}^{2}\right)$,

$$
\begin{aligned}
(\Gamma \varphi)(x, y)=Q_{\alpha} Q_{\beta} \varphi & (x-1, y-1)+Q_{\alpha} P_{\beta} \varphi(x-1, y+1) \\
& +P_{\alpha} Q_{\beta} \varphi(x+1, y-1)+P_{\alpha} P_{\beta} \varphi(x+1, y+1), \quad((x, y) \notin \partial V),
\end{aligned}
$$

and

$$
\begin{aligned}
(\Gamma \varphi)(x, y)=S_{\alpha} Q_{\beta} \varphi & (x, y-1)+S_{\alpha} P_{\beta} \varphi(x, y+1) \\
& +P_{\alpha} Q_{\beta} \varphi(x+1, y-1)+P_{\alpha} P_{\beta} \varphi(x+1, y+1), \quad((x, y) \in \partial V) .
\end{aligned}
$$

Then we have

$$
\left.U^{2}\right|_{\mathcal{A}^{(\leftrightarrow)}}=\mathcal{U}_{e}^{-1} \Gamma \mathcal{U}_{e}
$$


Proof. See Appendix B

The notion of the weights associated with the one-step move to the neighboring vertex; $P, Q$ and $S$, comes from a quantum analog of a discrete-time random walk [17] and becomes useful for the Fourier analysis in the next section. From now on, using Lemma 1, we will focus upon the unitary operator $\Gamma$ on $\ell^{2}\left(V ; \mathbb{C}^{2}\right)$ instead of the unitary operator $U$ on $\mathcal{A}$.

\section{Connecting with the CMV matrix}

In the previous section, the model is reduced to the quantum walk on $\ell^{2}\left(V ; \mathbb{C}^{2}\right)$ in which the walker moves to a neighboring location with the two-dimensional matrix weight following the unitary time evolution $\Gamma$. One of the keys to this paper is the connection between the AE model and the CMV matrix [9]. The spectral theory on the CMV matrix can be used to obtain the detailed spectral information, including gaps in the bulk spectrum and the edge state of our quantum walk model. To show this connection, we prepare the following two maps. Set $L^{2}\left(\mathbb{Z}_{+} \times[0,2 \pi) ; \mathbb{C}^{2}\right)$ by $\left\{\hat{\varphi}: \mathbb{Z}_{+} \times[0,2 \pi) \rightarrow \mathbb{C}^{2} \mid \sum_{j \in \mathbb{Z}_{+}} \int_{0}^{2 \pi}\|\hat{\varphi}(j ; k)\|_{\mathbb{C}^{2}}^{2} d k<\infty\right\}$.

Definition 2. Let $\mathcal{F}: \ell^{2}\left(V ; \mathbb{C}^{2}\right) \rightarrow L^{2}\left(\mathbb{Z}_{+} \times[0,2 \pi) ; \mathbb{C}^{2}\right)$ be the Fourier transform defined by

$$
\hat{\varphi}(j ; k):=(\mathcal{F} \varphi)(j ; k)=\sum_{m \in \mathbb{Z}} \varphi(j, m) e^{i k m}(j \in \mathbb{Z}, k \in[0,2 \pi)),
$$

and for fixed $k \in[0,2 \pi)$ we can write $\varphi^{\prime}(\cdot)=\hat{\varphi}(\cdot ; k)$.

Definition 3. For fixed $k \in[0,2 \pi)$, let $\Lambda_{k}: \ell^{2}\left(\mathbb{Z}_{+} ; \mathbb{C}^{2}\right) \rightarrow \ell^{2}\left(\mathbb{Z}_{+}\right)$be defined by

$$
\left(\Lambda_{k} \varphi^{\prime}\right)(j)=e^{i \omega(j)} \times \begin{cases}\left\langle 1 \mid \varphi^{\prime}([j / 2])\right\rangle & : j \text { is even }, \\ \left\langle 0 \mid \varphi^{\prime}([j / 2])\right\rangle & : j \text { is odd } .\end{cases}
$$

Here $[a]$ is the maximum integer such that $[a] \leq$ a for $a \in \mathbb{R}$ and

$$
\omega(2 j)=-j \arg \left(\left\langle 0\left|\hat{H}_{k}\right| 0\right\rangle\right), \omega(2 j+1)=(j+1) \arg \left(\left\langle 1\left|\hat{H}_{k}\right| 1\right\rangle\right),
$$

where $\hat{H}_{k} \in \mathrm{SU}(2)$ is

$$
\hat{H}_{k}=\left[\begin{array}{ll}
e^{-i k} \cos \alpha \cos \beta-e^{i k} \sin \alpha \sin \beta & -e^{-i k} \cos \alpha \sin \beta-e^{i k} \sin \alpha \cos \beta \\
e^{-i k} \sin \alpha \cos \beta+e^{i k} \cos \alpha \sin \beta & -e^{-i k} \sin \alpha \sin \beta+e^{i k} \cos \alpha \cos \beta
\end{array}\right] .
$$

Remark 2. The inverse maps of $\mathcal{F}^{-1}: L^{2}\left(\mathbb{Z}_{+} \times[0,2 \pi) ; \mathbb{C}^{2}\right) \rightarrow \ell^{2}\left(V ; \mathbb{C}^{2}\right)$ and $\Lambda_{k}^{-1}: \ell^{2}\left(\mathbb{Z}_{+}\right) \rightarrow$ $\ell^{2}\left(\mathbb{Z}_{+} ; \mathbb{C}^{2}\right)$ are

$$
\begin{gathered}
\left(\mathcal{F}^{-1} \hat{\varphi}\right)(x, y)=\int_{0}^{2 \pi} \hat{\varphi}(x ; k) e^{-i k y} \frac{d k}{2 \pi}, \\
\left(\Lambda_{k}^{-1} f\right)(j)=\left[\begin{array}{c}
e^{-i \omega(2 j+1)} f(2 j+1) \\
e^{-i \omega(2 j)} f(2 j)
\end{array}\right] .
\end{gathered}
$$

Remark 3. For every $\hat{\varphi} \in L^{2}\left(\mathbb{Z}_{+} \times[0,2 \pi) ; \mathbb{C}^{2}\right)$, by Fubini's theorem,

(1) $\Lambda_{k} \hat{\varphi}$ is well-defined, because $\hat{\varphi}(\cdot ; k)=\varphi^{\prime}(\cdot)$ belongs to $\ell^{2}\left(\mathbb{Z}_{+} ; \mathbb{C}^{2}\right)$ for fixed $k \in[0,2 \pi)$;

(2) for every unitary operator $E$ on $\ell^{2}\left(\mathbb{Z}_{+}\right)$, we have $\Lambda_{k}^{-1} E \Lambda_{k} \hat{\varphi}(\cdot ; k) \in \ell^{2}\left(V ; \mathbb{C}^{2}\right)$ for fixed $k$, and $\Lambda_{k}^{-1} E \Lambda_{k} \hat{\varphi} \in L^{2}\left(\mathbb{Z}_{+} \times[0,2 \pi) ; \mathbb{C}^{2}\right)$. 


\subsection{The CMV matrix}

We use $\mathbb{T}$ to denote the unit circle on the complex plane, that is, $\mathbb{T}=\{z \in \mathbb{C}|| z \mid=1\}$. For a given positive measure $\mu$ on $\mathbb{T}$, we set the Hilbert space $L_{\mu}^{2}(\mathbb{T})$ whose inner product is defined by

$$
\langle f, g\rangle_{\mu}=\int_{z \in \mathbb{T}} \overline{f(z)} g(z) d \mu(z) .
$$

Let $\left\{\chi_{j}(z)\right\}_{j=0}^{\infty} \subset L_{\mu}^{2}(\mathbb{D})$ be the orthogonal Laurent polynomials associated with the spectral measure $d \mu(z)$ on the unit circle in the complex plane obtained by the orthogonalization of $\left\{1, z, z^{-1}, z^{2}, \ldots\right\}$. The CMV matrix $\mathcal{C}$ is expressed by

$$
(\mathcal{C})_{i, j}=\left\langle\chi_{i}, z \chi_{j}\right\rangle_{\mu}
$$

which represents a canonical representation of the multiplication operation on $L_{\mu}^{2}(\mathbb{T}) ; f(z) \mapsto$ $z f(z)$. There is a one-to-one correspondence between the CMV matrix and so called Verblunsky parameters $\left(\eta_{0}, \eta_{1}, \eta_{2}, \ldots\right)$, which are complex parameters satisfying $\left|\eta_{j}\right|<1$. This correspondence is given in [7, 8]. The CMV matrix is expressed in full as

$$
\mathcal{C}=\left[\begin{array}{ccccccccc}
\bar{\eta}_{0} & \rho_{0} \bar{\eta}_{1} & \rho_{0} \rho_{1} & 0 & 0 & 0 & 0 & 0 & \ldots \\
\rho_{0} & -\eta_{0} \bar{\eta}_{1} & -\eta_{0} \rho_{1} & 0 & 0 & 0 & 0 & 0 & \ldots \\
0 & \rho_{1} \bar{\eta}_{2} & -\eta_{1} \bar{\eta}_{2} & \rho_{2} \bar{\eta}_{3} & \rho_{2} \rho_{3} & 0 & 0 & 0 & \ldots \\
0 & \rho_{1} \rho_{2} & -\eta_{1} \rho_{2} & -\eta_{2} \bar{\eta}_{3} & -\eta_{2} \rho_{3} & 0 & 0 & 0 & \ldots \\
0 & 0 & 0 & \rho_{3} \bar{\eta}_{4} & -\eta_{3} \bar{\eta}_{4} & \rho_{4} \bar{\eta}_{5} & \rho_{4} \rho_{5} & 0 & \ldots \\
0 & 0 & 0 & \rho_{3} \rho_{4} & -\eta_{3} \rho_{4} & -\eta_{4} \bar{\eta}_{5} & -\eta_{4} \rho_{5} & 0 & \ldots \\
& \vdots & \vdots & \vdots & \vdots & \vdots & \ddots & &
\end{array}\right],
$$

where $\rho_{j}=\sqrt{1-\left|\alpha_{j}\right|^{2}}$.

Now we are ready to state the following theorem which claims that the time evolution of the AE model converted to the vertex-based expression; $\Gamma=\left.\mathcal{U}_{e} U^{2}\right|_{\mathcal{A}(\leftrightarrow)} \mathcal{U}_{e}^{-1}$, is decomposed into the CMV matrices $\left\{\mathcal{C}_{k}\right\}_{k=0}^{2 \pi}$.

Theorem 1. Let $\mathcal{C}_{k}$ be the $C M V$ matrix whose Verblunsky parameters are $(\eta(k), 0, \eta(k), 0, \ldots)$, where $\eta(k)=\sin (\alpha-\beta) \cos k+i \sin (\alpha+\beta) \sin k$. Then we have

$$
\left(\Gamma^{n} \varphi\right)(x, y)=\int_{0}^{2 \pi}\left(\Lambda_{k}^{-1}\left({ }^{T} \mathcal{C}_{k}\right)^{n} \Lambda_{k} \hat{\varphi}\right)(x) e^{-i k y} \frac{d k}{2 \pi}, \quad((x, y) \in V) .
$$

The RHS of (3.1) is the $n$-th iteration value of the AE model at the vertex $(x, y) \in V$ with the initial state $\varphi \in \ell^{2}\left(V ; \mathbb{C}^{2}\right)$, On the other hand, the integral on the LHS consists of the $2 x+1$ and $2 x$ components with the phase rotations $e^{-i(\omega(2 x+1)+k y)}$ and $e^{-i(\omega(2 x)+k y)}$, respectively, of the $n$th composition of the CMV matrix ${ }^{T} \mathcal{C}_{k}$ with the initial state $\Lambda_{k} \hat{\varphi} \in$ $\ell^{2}\left(\mathbb{Z}_{+}\right)$. Thus the problem is reduced to the analysis on the RHS of (3.1), using well developed studies on the spectral analysis on the CMV matrix to obtain spectral information about this walk. 


\subsection{Reduction to a quantum walk on the half line: Type-I quan- tum walk}

Due to the translation invariance to the $y$-axis direction of the AE model, we perform the partial Fourier transform defined in the previous subsection. We will show that the AE model is decomposed into a wave number dependent quantum walk on the half line. This reduced quantum walk is given by a vertex-based expression. For fixed $k \in[0,2 \pi)$, we define $\hat{\Gamma}_{k}: \ell^{2}\left(\mathbb{Z} ; \mathbb{C}^{2}\right) \rightarrow \ell^{2}\left(\mathbb{Z} ; \mathbb{C}^{2}\right)$ by

$$
\left(\hat{\Gamma}_{k} \varphi^{\prime}\right)(x)= \begin{cases}\hat{P}_{k} \varphi^{\prime}(x+1)+\hat{Q}_{k} \varphi^{\prime}(x-1) & : x \geq 1, \\ \hat{P}_{k} \varphi^{\prime}(x+1)+\hat{S}_{k} \varphi^{\prime}(x) & : x=0\end{cases}
$$

where $\hat{P}_{k}=|0\rangle\left\langle 0\left|\hat{H}_{k}, \hat{Q}_{k}=\right| 1\right\rangle\langle 1| \hat{H}_{k}$ and $\hat{S}_{k}=|1\rangle\langle 0| \hat{H}_{k}$. Here $\hat{H}_{k}$ is defined by $H_{\alpha} \hat{D}(k) H_{\beta}$, where

$$
\hat{D}_{k}=\left[\begin{array}{cc}
e^{-i k} & 0 \\
0 & e^{i k}
\end{array}\right] \text {. }
$$

More precisely,

$$
\hat{H}_{k}=\left[\begin{array}{ll}
e^{-i k} \cos \alpha \cos \beta-e^{i k} \sin \alpha \sin \beta & -e^{-i k} \cos \alpha \sin \beta-e^{i k} \sin \alpha \cos \beta \\
e^{-i k} \sin \alpha \cos \beta+e^{i k} \cos \alpha \sin \beta & -e^{-i k} \sin \alpha \sin \beta+e^{i k} \cos \alpha \cos \beta
\end{array}\right] .
$$

Then we have the following lemma.

Lemma 3. Let $\varphi_{n} \in \ell^{2}\left(V ; \mathbb{C}^{2}\right)$ be the $n$-th iteration of $\Gamma$ with the initial state $\varphi_{0}(\boldsymbol{x})=$ $\delta(\boldsymbol{x})^{T}[0,1]$, that is, $\varphi_{n}=\Gamma^{n} \varphi_{0}$. The Fourier transform of $\varphi_{n}$ described by $\mathcal{F}\left(\varphi_{n}\right)=\hat{\varphi}_{n} \in$ $L^{2}\left(\mathbb{Z} \times[0,2 \pi) ; \mathbb{C}^{2}\right)$ is expressed by

$$
\hat{\varphi}_{n}=\hat{\Gamma}_{k}^{n} \hat{\varphi}_{0}, \hat{\varphi}_{0}=\delta(x)^{T}[0,1] .
$$

Proof. By the shift invariance with respect to the vertical direction and Lemma 2, we immediately prove this lemma.

The connection between the CMV matrix and the quantum walk was first shown in [9]. The Type-I quantum walk called by [25] is a quantum walk on the line induced by a special choice of the Verblunsky parameters of the CMV matrix. The Type-I quantum walk is defined by the arc-based expression as is the AE model's arc-based expression in Sect. 2.2 . Thus we prepare the notation for the arcs on the half line. Let $A^{\prime}$ be the arc set of $\mathbb{Z}_{+}$with the self-loop at the origin, that is,

$$
A^{\prime}=\left\{(x ; 0),(x ; 1) \mid x \in \mathbb{Z}_{+}\right\} .
$$

Here $(x ; 0)$ indicates the arc from $x+1$ to $x$, and $(x ; 1)$ indicates the arc from $x-1$ to $x$ for $x \geq 1$, and the self-loop for $x=0$. We define the subsets of $A^{\prime}$ by

$$
A_{x}^{\prime}=\{(x ; 0),(x ; 1)\}
$$

for each $x \in \mathbb{Z}_{+}$. 
Definition 4. The Type-I quantum walk [9, 25] is defined as follows:

(1) Total state space: $\mathcal{A}^{\prime}=\ell^{2}\left(A^{\prime}\right)$

(2) Time evolution: We set $\mathcal{A}_{x}^{\prime}=\left\{\varphi^{\prime} \in \mathcal{A}^{\prime} \mid a \notin A_{x} \Rightarrow \varphi^{\prime}(a)=0\right\}$. The local unitary operator on $\mathcal{A}_{j}^{\prime}$ is defined by

$$
C_{x}^{\prime} \cong\left[\begin{array}{ll}
\gamma & \delta \\
\alpha & \beta
\end{array}\right]
$$

setting the canonical basis of $\mathcal{A}_{x}^{\prime}$ by $\left\{\delta_{(x ; 0)}, \delta_{(x ; 1)}\right\}$ in this order. The time evolution $W: \mathcal{A}^{\prime} \rightarrow \mathcal{A}^{\prime}$ is described by

$$
(W \psi)(a)=\sum_{b: t(a)=o(b)}\left\langle\delta_{\bar{a}}, C_{x}^{\prime} \delta_{b}\right\rangle \psi(b) \text { for } a \in A^{\prime} \text { with } o(a)=x .
$$

As in the previous section, we also define $\mathcal{U}^{\prime}: \ell^{2}\left(A^{\prime}\right) \rightarrow \ell^{2}\left(\mathbb{Z}_{+} ; \mathbb{C}^{2}\right)$ as follows:

$$
\left(\mathcal{U}^{\prime} \psi\right)(x)={ }^{T}[\psi(x ; 0), \psi(x ; 1)](x \in \mathbb{Z}, j \in\{0,1\}) .
$$

The inverse map is $\left(\mathcal{U}^{\prime-1} \varphi\right)(x ; j)=\varphi_{j}(x)$. Putting $\Gamma^{\prime}=\mathcal{U}^{\prime} W \mathcal{U}^{\prime-1}$, we take the $n$-th iteration of $\Gamma^{\prime}$ as $\varphi_{n}^{\prime}$ with the initial state $\varphi_{0}^{\prime}$, that is, $\varphi_{n}^{\prime}=\Gamma^{\prime n} \varphi_{0}$. From simple observation, we have

$$
\varphi_{n+1}^{\prime}(x)= \begin{cases}Q \varphi_{n}^{\prime}(x-1)+P \varphi_{n}^{\prime}(x+1) & : x \geq 1, \\ S \varphi_{n}^{\prime}(x)+P \varphi_{n}^{\prime}(x+1) & : x=0 .\end{cases}
$$

Here $P=|0\rangle\langle 0|H, Q=| 1\rangle\langle 1| H$ and $S=|0\rangle\langle 1| H$ with

$$
H=\left[\begin{array}{ll}
\alpha & \beta \\
\gamma & \delta
\end{array}\right] \in \mathrm{U}(2)
$$

Therefore, $\mathcal{U}^{\prime} \hat{\Gamma}_{k} \mathcal{U}^{\prime-1}$ is identical to the time evolution of the Type-I quantum walk in the case of $H=\hat{H}_{k}$ for fixed $k$. Mapping the time iteration of $\hat{\Gamma}_{k}$ to that of Type-I quantum walk has the following benefit.

Lemma 4. [9, 25] For a given Type-I quantum walk with the local unitary coin $C_{j}^{\prime}$ (3.5), let $\mathcal{D}: \mathcal{A}^{\prime} \rightarrow \ell^{2}\left(\mathbb{Z}_{+}\right)$b屯

$$
(\mathcal{D} \psi)(m)= \begin{cases}\psi((m+1) / 2 ; 0) e^{i \arg (a)(m+1) / 2} & : m \text { is odd } \\ \psi(m / 2 ; 1) e^{-i \arg (d) m / 2} & : m \text { is even }\end{cases}
$$

${ }^{*}$ The inverse map $\mathcal{D}^{-1}: \ell^{2}\left(\mathbb{Z}_{+}\right) \rightarrow \mathcal{A}^{\prime}$ is

$$
(\mathcal{D} f)(m ; j)= \begin{cases}f(2 m+1) e^{-i(2 m+1) \arg (a)} & : j=0 \\ f(2 m) e^{i 2 m a r g(d)} & : j=1\end{cases}
$$


The time evolution of the Type-I quantum walk, $W$, is unitarily equivalent to the CMV matrix with null odd Verblunsky parameter $\left(\eta_{0}, 0, \eta_{2}, 0, \eta_{4}, 0, \ldots\right)$, that is,

$$
W=\mathcal{D}^{-1 T} \mathcal{C} \mathcal{D}
$$

where

$$
\eta_{j}= \begin{cases}\eta \Delta^{-(j+1) / 2} & : j \text { is even } \\ 0 & : \text { otherwise }\end{cases}
$$

with $\eta=\Delta^{1 / 2} \bar{\beta}, \Delta=\alpha \beta-\gamma \delta$.

Now we are ready to prove Theorem 1.

\section{Proof of Theorem 1}

Since $\operatorname{det}\left(H_{\alpha}\right)=\operatorname{det}\left(H_{\beta}\right)=\operatorname{det}\left(\hat{D}_{k}\right)=1$, we have $\Delta=\operatorname{det}\left(\hat{H}_{k}\right)=1$. Thus concerning our local quantum coin (3.3), the corresponding Verblunsky parameter is $\eta_{j}=\eta(k)$ ( $j$ is even), where

$$
\eta(k)=\overline{\left\langle 0\left|\hat{H}_{k}\right| 1\right\rangle}=-\cos k \sin (\alpha+\beta)+i \sin k \sin (\alpha-\beta) .
$$

By Lemma 4 ,

$$
\mathcal{U}^{\prime} \hat{\Gamma}_{k} \mathcal{U}^{\prime-1}=\mathcal{D}^{-1}{ }^{T} \mathcal{C}_{k} \mathcal{D}
$$

Remarking that $\Lambda_{k}=\mathcal{D} \mathcal{U}^{\prime}$, we have

$$
\Lambda_{k}^{-1 T} \mathcal{C}_{k}^{n} \Lambda_{k} \hat{\varphi}_{0}=\hat{\varphi}_{n}
$$

Taking the Fourier inverse of the above, that is,

$$
\varphi(x, y)=\int_{0}^{2 \pi} \hat{\varphi}_{n}(x ; k) e^{-i k y} \frac{d k}{2 \pi},
$$

we obtain the desired conclusion.

\section{Spectrum, Dispersion Relation, and Topological Phases}

In this section, we study spectral properties of the quantum walks $\hat{\Gamma}_{k}$ and compare them with the result by topological phases which are independently studied from the quantum walk without boundaries. In order to connect with both results, we focus on the wave number dependence's of the spectrum, which is called dispersion relations in the language of physics. We see that the location of spectrum agree well with the result by topological numbers.

\subsection{Spectrum and dispersion relation of the AE model}

In the previous section, we proved the decomposition of the time evolution of the AE model $\Gamma$ into the wave number dependent quantum walk on the half line $\hat{\Gamma}_{k}$. Furthermore it is shown that this quantum walk $\hat{\Gamma}_{k}$ is co-spectral to the CMV matrix $\mathcal{C}_{k}$. Thus the spectral analysis on the CMV matrix for fixed $k$ can be used to get the spectral information about the AE model. Indeed we will provide a quite explicit formula for the spectrum of $\hat{\Gamma}_{k}$ by the spectrum $\sigma\left(\mathcal{C}_{k}\right)$ of $\mathcal{C}_{k}$. We, furthermore, re-express this result in terms of physics, 
i.e., the dispersion relation, which clarifies the wave number dependence of the spectrum. Actually the dispersion relation is described by nothing but the subset of $[0,2 \pi)^{2}$ such that $\left\{(k, \theta) \mid e^{i \theta} \in \sigma\left(\mathcal{C}_{k}\right), k \in[0,2 \pi)\right\}$.

Now let us define some important functions to describe the above statement more explicitly:

$$
\begin{aligned}
\rho(k) & :=\sqrt{1-|\eta(k)|^{2}}=\sqrt{\cos ^{2}(\alpha-\beta)-\sin 2 \alpha \sin 2 \beta \cos ^{2} k}, \\
m_{0}(k) & :=m_{0}= \begin{cases}\frac{|\cos k \sin (\alpha+\beta)|}{\sqrt{1-\sin ^{2}(\alpha-\beta) \sin ^{2} k}} \quad: \rho(k) \neq 0, \\
1 \quad: \rho(k)=0,\end{cases} \\
\theta_{0}(k) & := \begin{cases}\arcsin (-\sin k \sin (\alpha-\beta)) & : \cos k \sin (\alpha+\beta) \leq 0, \\
\pi-\arcsin (-\sin k \sin (\alpha-\beta)) & : \cos k \sin (\alpha+\beta)>0 .\end{cases}
\end{aligned}
$$

We obtain a detailed expression for the spectrum of $\mathcal{C}_{k}$ using the above values as follows.

Lemma 5. For fixed $k \in[0,2 \pi)$, the spectrum of $\mathcal{C}_{k}$ is decomposed into continuous spectrum $\sigma_{c}^{(k)}$ and point spectrum $\sigma_{p}^{(k)}$, that is, $\sigma\left(\mathcal{C}_{k}\right)=\sigma_{c}^{(k)} \sqcup \sigma_{p}^{(k)}$, where

$$
\begin{aligned}
\sigma_{c}^{(k)} & =\left\{e^{i \theta}|| \cos \theta \mid \leq \rho(k)\right\} \\
\sigma_{p}^{(k)} & = \begin{cases}\left\{e^{i \theta_{0}}(k)\right\} & : \sin (\alpha-\beta) \neq 0, k \notin\{\pi / 2,3 \pi / 2\}, \\
\emptyset & : \text { otherwise. }\end{cases}
\end{aligned}
$$

Moreover we have

$$
\sigma\left(\left.U^{2}\right|_{\mathcal{A}^{(\leftrightarrow)}}\right)=\bigcup_{k \in[0,2 \pi)}\left(\sigma_{c}^{(k)} \sqcup \sigma_{p}^{(k)}\right) .
$$

Proof. The detailed spectrum of this CMV matrix has already been obtained by [9, 25]. We summarize partially the results as follows.

Lemma 6. [9, 25] The spectral measure of the CMV matrix with the Verblunsky parameter $(\eta, 0, \eta, 0, \ldots)$ is described as follows.

(1) For $|\eta|=1$ case.

$$
d \mu(\theta)=\delta(\theta+\arccos (\eta))
$$

Here $\delta(\cdot)$ is the Dirac delta function on $[0,2 \pi)$

(2) For $|\eta|<1$ case.

$$
d \mu(\theta)=w(\theta) \frac{d \theta}{2 \pi}+m_{0} \delta\left(\theta-\theta_{0}\right)
$$

Here $w(\theta)$ is the weight of the absolutely continuous part, given by

$$
w(\theta)=\frac{\sqrt{\rho^{2}-\cos ^{2} \theta}}{|\sin \theta+\operatorname{Im}(\eta)|} \mathbf{1}_{\{|\cos \theta|<\rho\}}(\theta), \quad\left(\rho=\sqrt{1-|\eta|^{2}}\right)
$$

and $m_{0} \in[0,1]$ is the mass at $\theta_{0} \in[0,2 \pi)$, where

$$
\begin{gathered}
m_{0}=\frac{|\operatorname{Re}(\eta)|}{\sqrt{1-\operatorname{Im}^{2}(\eta)}}, \\
\theta_{0}= \begin{cases}\arcsin (-\operatorname{Im}(\eta)) & : \operatorname{Re}(\eta) \geq 0 \\
\pi-\arcsin (-\operatorname{Im}(\eta)) & : \operatorname{Re}(\eta)<0 .\end{cases}
\end{gathered}
$$


Thus Lemma 6 immediately implies the conclusion by replacing $\eta$ into $\eta=\eta(k)$.

Defining $\theta_{c}(k)$ as $\arccos (\rho(k))$, that is,

$$
\theta_{c}(k)=\arccos \left(\sqrt{\cos ^{2}(\alpha-\beta)-\sin 2 \alpha \sin 2 \beta \cos ^{2} k}\right),
$$

we find that the continuous spectrum of $\mathcal{C}_{k}$ is $\left\{e^{i \theta} \mid \theta \in\left[\theta_{c}(k), \pi-\theta_{c}(k)\right] \cup\left[\pi+\theta_{c}(k), 2 \pi-\right.\right.$ $\left.\left.\theta_{c}(k)\right]\right\}$, and the point spectrum is $\left\{\theta_{0}(k)\right\}$. In the language of physics, an argument of a spectrum of unitary time-evolution operators is called quasi-energy, which is a main reason to re-express $\sigma_{c}^{(k)}$ and $\sigma_{p}^{(k)}$ by quasi-energy $\theta_{c}$ in (4.12) and $\theta_{0}$ in (4.3), respectively. We call $\left[\theta_{c}(k), \pi-\theta_{c}(k)\right] \cup\left[\pi+\theta_{c}(k), 2 \pi-\theta_{c}(k)\right]$ and $\left\{\theta_{0}\right\}$ the bulk spectrum and the edge spectrum. These names come from the fact that the bulk spectrum corresponds to the spectrum of walker's states in absence of any boundary (cutting edge of systems), while the point spectrum is a consequence of the presence of the cutting edge. As we show in section 4.2, the existence of edge spectrum is consistent with a result by the bulk-edge correspondence from a topological properties of the bulk states. Furthermore, we will show that states for the edge spectrum exponential localizes around the cutting edge in Section 6 . Particularly, the wave number $k$ dependence of (quasi-)energy $\theta$ is called dispersion relation. Thereby, Bu and $E d$ defined by (4.13) and (4.14) in the following definition can be regarded as the dispersion relations.

Definition 5. Let $\sigma_{c}^{(k)}$ and $\sigma_{p}^{(k)}$ be the continuous and point spectrum's of $\mathcal{C}_{k}$ for fixed $k$. Then we set

$$
\begin{aligned}
& B u=\bigcup_{k \in[0,2 \pi)}\left\{(k, \theta) \mid e^{i \theta} \in \sigma_{c}^{(k)}\right\}, \\
& E d=\bigcup_{k \in[0,2 \pi)}\left\{(k, \theta) \mid e^{i \theta} \in \sigma_{p}^{(k)}\right\} .
\end{aligned}
$$

In our system, the dispersion relation is decomposed into $B u$ and $E d, B u \cup E d$, and we call $B u$ and $E d$ dispersion relations for bulk and edge states, respectively. The dispersion relation provides important information for group and phase velocities, topological numbers, and so on. By Lemma 5, we obtain quite explicit form of the continuous spectrum $\sigma_{c}^{(k)}$ and the point spectrum $\sigma_{p}^{(k)}$ of the wave number dependent quantum walk on the half line $\hat{\Gamma}_{k}$ whose spectrum is equivalent to $\sigma\left(\mathcal{C}_{k}\right)$. The dispersion relation for bulk and edge states, $B u$ and $E d$, are described by $\sigma_{c}^{(k)}$ and $\sigma_{p}^{(k)}$. Therefore we can also provide explicit expressions for $B u$ and $E d \subset[0,2 \pi)^{2}$ as follows.

Theorem 2. Let $\theta_{0}(k)$ and $\theta_{c}(k)$ be the above defined in (4.3) and (4.12), respectively. Then we have

$$
\begin{aligned}
& B u=\bigcup_{k \in[0,2 \pi)}\left[\theta_{c}(k), \pi-\theta_{c}(k)\right] \cup\left[\pi+\theta_{c}(k), 2 \pi-\theta_{c}(k)\right] \\
& E d= \begin{cases}\left\{\left(k, \theta_{0}(k)\right) \mid k \in[0,2 \pi) \backslash\{\pi / 2,3 \pi / 2\}\right\} & : \sin (\alpha-\beta) \neq 0, \\
\emptyset & : \sin (\alpha-\beta)=0,\end{cases}
\end{aligned}
$$


By (4.15), we can identify the condition for the parameters $(\alpha, \beta)$ of the AE model so that there are no gaps in the bulk spectrum.

Corollary 1. Let $\theta_{0}(k)$ and $\theta_{c}(k)$ be the above defined in (4.3) and 4.12), respectively. There exists wave number $k \in[0,2 \pi)$ at which gaps in the bulk spectrum vanish if and only if $" \sin (\alpha-\beta)=0$ and $\sin 2 \alpha \sin 2 \beta \leq 0$ " or $" \sin (\alpha+\beta)=0$ and $\sin 2 \alpha \sin 2 \beta>0$ ". In the first parameter class, the wave numbers are $k=\pi / 2$ and $3 \pi / 2$, in the second parameter class, ones are $k=0$ and $\pi$.

We define $\theta_{0}(k)$ if the mass point $m_{0}(k)$ is strictly positive. Concerning the value of the mass point $m_{0}(k)$ described by (4.2), the parameter $(\alpha, \beta)$ must satisfy $\sin (\alpha+\beta) \neq 0$ and under this restriction, we set the domain of $\theta_{0}(k)$ as $[0,2 \pi) \backslash\{\pi / 2,3 \pi / 2\}$. Under this setting of the parameter and the domain, we call $\theta_{0}(k)$ the quasi-energy for edge states or the edge spectrum, and $E d$ the dispersion relation for edge states. Furthermore, the derivative of the quasi-energy for edge states $\theta_{0}(k)$ is called the group velocity for the edge state. (Since we focus on the group velocity only for the edge states in the present work, we omit "for the edge states" hereafter to use the term.) The group velocity corresponds to the velocity of the envelope of a wave packet, as we will explain in section 5 .

Corollary 2. The dispersion relation for edge states is monotonic on $\mathbb{T}:=\mathbb{R} /(2 \pi \mathbb{Z})$ with $E d \cap B u=\emptyset$, and is discontinuous only at $k=\pi / 2$ and $3 \pi / 2 \bmod (2 \pi)$ on $\mathbb{T}$. At these points there is a jump, that is,

$$
\lim _{k \uparrow \pi / 2} \theta_{0}(k) \neq \lim _{k \downarrow \pi / 2} \theta_{0}(k), \quad \lim _{k \uparrow 3 \pi / 2} \theta_{0}(k) \neq \lim _{k \downarrow 3 \pi / 2} \theta_{0}(k),
$$

and

$$
\lim _{k \uparrow \pi / 2,3 \pi / 2} \theta_{0}(k), \lim _{k \downarrow \pi / 2,3 \pi / 2} \theta_{0}(k) \in B u .
$$

This relation explains why we relate $\theta_{0}$ and Ed with edge states, since it is well known in the research field of topological phases of matter that edge states originating from topological phases should appear in gaps of the bulk spectrum so that the edge spectrum connects two endpoints of the different interval of the bulk spectrum. Furthermore, in section 6, we provide a clear evidence that states with $\theta_{0}$ exponentially localize near self-loops on the boundary by deriving the limit distribution towards bulk.

More precisely, we divide the space of parameters $(\alpha, \beta) \in[0,2 \pi)^{2}$ into six classes according to the pair of signs; $(\operatorname{sgn}(\sin (\alpha+\beta)), \operatorname{sgn}(\sin (\alpha-\beta)))$, as follows:

(1) $(0,0)$ and $(0, \pm)$ classes:

This is the condition for vanishing gaps in the bulk spectrum at $k=0$ and $k=\pi$. There are no edge states for all $k \in[0,2 \pi)$, that is, $\theta_{0}(k)$ cannot be defined.

(2) $(+,+)$ class:

The dispersion relation for edge states monotonically increases on $\mathbb{T}$. The discontinuity at $k=\pi / 2$ occurs between " $\left(\pi / 2, \pi+\theta_{c}(\pi / 2)\right)$ and $\left(\pi / 2,2 \pi-\theta_{c}(\pi / 2)\right)$ " $\in[0,2 \pi)^{2}$ and the one at $k=3 \pi / 2$ occurs between " $\left(3 \pi / 2, \theta_{c}(3 \pi / 2)\right)$ and $\left(\pi / 2, \pi-\theta_{c}(3 \pi / 2)\right)$ " $\in[0,2 \pi)^{2}$, respectively. 
(3) $(+,-)$ class:

The dispersion relation for edge states monotonically decreases on $\mathbb{T}$. The discontinuity at $k=\pi / 2$ occurs between " $\left(\pi / 2, \theta_{c}(\pi / 2)\right)$ and $\left(\pi / 2, \pi-\theta_{c}(\pi / 2)\right)$ " $\in[0,2 \pi)^{2}$ and that at $k=3 \pi / 2$ occurs between " $\left(3 \pi / 2, \pi+\theta_{c}(3 \pi / 2)\right)$ and $\left(\pi / 2,2 \pi-\theta_{c}(3 \pi / 2)\right)$ " $\in[0,2 \pi)^{2}$, respectively.

(4) $(-,+)$ class:

The dispersion relation for edge states monotonically decreases on $\mathbb{T}$. The discontinuity at $k=\pi / 2$ occurs between " $\left(\pi / 2, \pi+\theta_{c}(\pi / 2)\right)$ and $\left(\pi / 2,2 \pi-\theta_{c}(\pi / 2)\right)$ " $\in[0,2 \pi)^{2}$ and that at $k=3 \pi / 2$ occurs between " $\left(3 \pi / 2, \pi+\theta_{c}(3 \pi / 2)\right)$ and $\left(\pi / 2,2 \pi-\theta_{c}(3 \pi / 2)\right)$ " $\in$ $[0,2 \pi)^{2}$, respectively.

(5) $(-,-)$ class:

The dispersion relation for edge states monotonically increases on $\mathbb{T}$. The discontinuity at $k=\pi / 2$ occurs between " $\left(\pi / 2, \theta_{c}(\pi / 2)\right)$ and $\left(\pi / 2, \pi-\theta_{c}(\pi / 2)\right)$ " $\in[0,2 \pi)^{2}$ and that at $k=3 \pi / 2$ occurs between " $\left(3 \pi / 2, \pi+\theta_{c}(3 \pi / 2)\right)$ and $\left(\pi / 2,2 \pi-\theta_{c}(3 \pi / 2)\right)$ " $\in[0,2 \pi)^{2}$, respectively.

(6) $( \pm, 0)$ class:

This is the condition for vanishing gaps in the bulk spectrum at $k=\pi / 2$ and $k=3 \pi / 2$.

The group velocity is zero, that is, the quasi-energy for the edge states has no wave number dependence.

By the above corollaries, the pattern shape of the dispersion relation $B u \cup E d$ is determined by the signs of $\sin 2 \alpha \sin 2 \beta=\sin ^{2}(\alpha+\beta)-\sin ^{2}(\alpha-\beta), \sin (\alpha+\beta)$ and $\sin (\alpha-\beta)$. See Figure 2. We set the triple of the signs $\left(\epsilon_{1}, \epsilon_{2}, \epsilon_{3}\right),\left(\epsilon_{j} \in\{ \pm\}\right)$, where $\epsilon_{1}=\operatorname{sgn}(\sin 2 \alpha \sin 2 \beta)$, $\epsilon_{2}=\operatorname{sgn}\left(\sin (\alpha+\beta)\right.$ and $\epsilon_{3}=\operatorname{sgn}(\sin (\alpha-\beta))$. The first sign provides the shape of $B u$ : we have two bands which are symmetric with respect to $\theta=\pi$, and each band has period $\pi$; if $\epsilon_{1}=+$, then "peaks" appear at $k=\pi / 2,3 \pi / 2$ and "troughs" appear at $k=0, \pi$ while if $\epsilon_{1}=-$, then we have the $\pi$-phase difference of the $\epsilon_{1}=+$ case. If $\sin (\alpha+\beta) \neq 0$, the edge state appears as a bridge connecting the peaks (resp. troughs) of the upper band and the lower band for $\epsilon_{1}=+$ case (resp. $\epsilon_{1}=-$ ). The signs $\epsilon_{2}$ and $\epsilon_{3}$ describe the pattern shape of the edge state. If $\epsilon_{2}=+$, then $\theta_{0}(0)=\pi$ and $\theta_{0}(\pi)=0(=2 \pi)$, while if $\epsilon_{2}=-$, then we have the $\pi$-phase shift of $\epsilon_{2}=+$ case. If $\epsilon_{2} \epsilon_{3}=+$, then the group velocity is positive, while if $\epsilon_{2} \epsilon_{3}=-$, then the group velocity is negative.

\subsection{Topological phases}

Here, we study symmetry and topological phases of the AE model. At first, we review basic notions of topological phases, then explain symmetry and topological numbers of the AE model studied in [4]. We remark that the topological number of the AE model derived in [4] is well-defined only when gaps in the bulk spectrum exist. As we will explain later, the result by obtained using the topological phases agrees with the presence or absence of edge states by Corollary 2 (1)-(5), but not Corollary 2 (6). This is because Corollary 2 (6) requires the absence of gaps in the bulk spectrum, where the topological numbers in [4] are not well-defined. We solve this problem by introducing a new topological number of the AE model, which is defined only when there are no gaps in the bulk spectrum. 
(a)

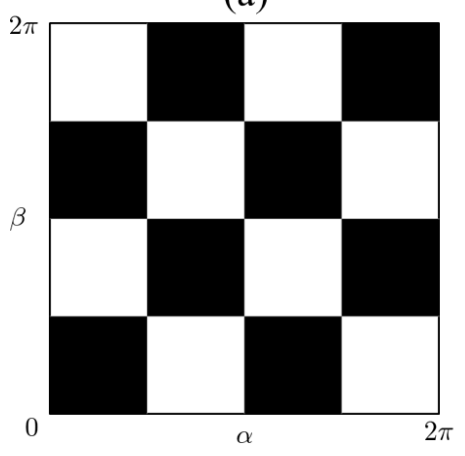

(b)

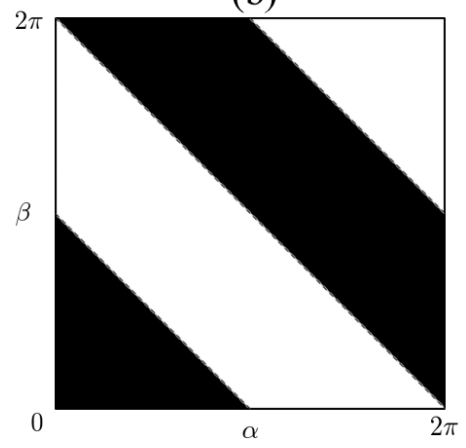

(c)

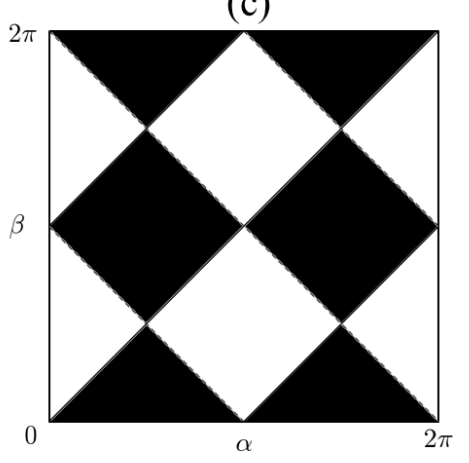

Figure 2: The black regions of $[0,2 \pi)^{2}$ depict (a) $\epsilon_{1}=\sin 2 \alpha \sin 2 \beta>0$ (b) $\epsilon_{2}=\sin (\alpha+\beta)>0$ (c) $\epsilon_{2} \epsilon_{3}=\sin (\alpha+\beta) \sin (\alpha-\beta)>0$, respectively. On the lines $\alpha+\beta=\pi, 2 \pi, 3 \pi$ (see i.g., (b)), the edge state does not appear.

Recently, localization appearing in space-inhomogeneous quantum walks has attracted attention, since it has been related to topological phase. Here, the topological numbers originating from non-trivial topological properties of the eigen functions in wavenumber space [36] are related to fundamental objects of study in algebraic topology, such as winding number or Chern number. This approach has emerged from recent research of topological phases of matter (known as topological insulators and superconductors) in condensed-matter physics [6, 12, 19, 31]. Since a quantum walk can be considered as a simplified theoretical model of a topological insulator (more preciously, Floquet topological insulators 21, 27, since quantum walks can be regarded as time-periodically driven systems), the topological phases of quantum walks in inhomogeneous systems have been intensively studied 2, 10, 11, 13, 14, 29, 28, In the field of theoretical physics of the topological insulators, a fundamental principle, the so called bulk-edge correspondence, states that the number of edge states localized at the interface between two adjacent regions in position space is the same as the difference in topological number between the two regions.

Now, we briefly introduce results concerning topological numbers in [4]. Since topological phases of matter are easier to consider for systems with translation symmetry (though this is not necessary for topological insulators), we focus on a system without boundaries and explain topological numbers originating from non-trivial topological properties of eigen functions in the wavenumber space. Accordingly, we consider the time-evolution operator $\Gamma$ in (2.5) for the vertex set

$$
V_{0}=\mathbb{Z} \times \mathbb{Z}=\{(x, y) \mid x \in \mathbb{Z}, y \in \mathbb{Z}\},
$$

in this section. Unlike (3.2), in applying the Fourier transform to the two-dimensional space of $\Gamma$, we derive the time-evolution operator in the wavenumber representation $\hat{\Gamma}_{0}\left(k_{x}, k_{y}\right)$ : $\mathbb{C}^{2} \rightarrow \mathbb{C}^{2}$ for fixed $\left(k_{x}, k_{y}\right) \in[0,2 \pi)^{2}$ described as

$$
\hat{\Gamma}_{0}\left(k_{x}, k_{y}\right)=\hat{D}\left(k_{x}\right) H_{\alpha} \hat{D}\left(k_{y}\right) H_{\beta} .
$$


We note that $\hat{D}(k)$ and $H_{\gamma}$ can be written as

$$
\begin{aligned}
\hat{D}(k) & =e^{-i k \sigma_{3}}, \\
H_{\gamma} & =e^{-i \gamma \sigma_{2}},
\end{aligned}
$$

respectively, using an identity matrix $\sigma_{0}$ and Pauli matrices $\sigma_{i}(i=1,2,3)$

$$
\sigma_{0}=\left[\begin{array}{ll}
1 & 0 \\
0 & 1
\end{array}\right], \sigma_{1}=\left[\begin{array}{ll}
0 & 1 \\
1 & 0
\end{array}\right], \sigma_{2}=\left[\begin{array}{cc}
0 & -i \\
i & 0
\end{array}\right], \sigma_{3}=\left[\begin{array}{cc}
1 & 0 \\
0 & -1
\end{array}\right] .
$$

First, we identify the symmetries of the time-evolution operator, $\hat{\Gamma}_{0}\left(k_{x}, k_{y}\right)$. It is well known that there are three important symmetries for topological phases, namely, timereversal, particle-hole, and chiral symmetries [6, 12, 19, 31]. These symmetries require the time-evolution operator to satisfy the following relations with anti-linear symmetry operators $\hat{T}$ and $\hat{P}$, and a linear symmetry operator $\hat{Y}$ (see details in [13]):

$$
\begin{aligned}
\text { Time-reversal symmetry: } & \hat{T} \hat{\Gamma}_{0}\left(k_{x}, k_{y}\right) \hat{T}^{-1}=\hat{\Gamma}_{0}\left(-k_{x},-k_{y}\right)^{-1}, \\
\text { Particle-hole symmetry: } & \hat{P} \hat{\Gamma}_{0}\left(k_{x}, k_{y}\right) \hat{P}^{-1}=\hat{\Gamma}_{0}\left(-k_{x},-k_{y}\right), \\
\text { Chiral symmetry: } & \hat{Y} \hat{\Gamma}_{0}\left(k_{x}, k_{y}\right) \hat{Y}^{-1}=\hat{\Gamma}_{0}\left(k_{x}, k_{y}\right)^{-1},
\end{aligned}
$$

On one hand, we see from (4.17) and (4.19) that particle-hole symmetry is retained with the symmetry operator

$$
\hat{P}=K,
$$

where $K$ stands for the complex conjugation. On the other hand, time-reversal and chiral symmetries are not generally satisfied. Therefore, the AE model belongs to class D in the classification table of topological phases 33 .

Roughly speaking, topological phases of matter are characterized by topological properties of wavefunctions, which are periodic functions in wavenumber space. The topological number $\nu_{2 d}$ for the AE model derived in Ref. [4] is obtained from a winding number originating from the periodicity of two wavenumber space $k_{x}$ and $k_{y}$ in addition to the time direction due to the periodic-time drive of quantum walks [32]. The topological number $\nu_{2 d}$ 's dependence on $a$ and $b$ is summarized in figure 3 (a). We note that the topological number $\nu_{2 d}$ is finite except on lines in figure 3 (a), where there are no gaps in the bulk spectrum and $\nu_{2 d}$ is not well-defined. Along with the bulk-edge correspondence, we can predict the emergence of non-degenerated edge states in the AE model when the topological number $\nu_{2 d}$ assigned to the parameter set $(\alpha, \beta)$ is non-zero, since in the outer region from the cutting edge of the AE model, $\nu_{2 d}$ is zero. Since the topological number $\nu_{2 d}$ is finite whenever there exist gaps in bulk spectrum. the phase diagram seems to agree with Corollary 2 (1)-(5).

Corollary 2 (6), however, states that edge states appear despite the fact that there are no gaps in the bulk spectrum and the topological number is not well-defined. This disagreement can be resolved by introducing a topological number that is calculated from $\hat{\Gamma}_{0}\left(k_{x}, k_{y}\right)$ with fixed wave-number $k_{y}$ such that gaps in the spectrum in the subspace $k_{x} \in[0,2 \pi)$ exist [35]. Therefore, the new topological number is similar to one defined in one dimension, as previously studied[3]. Remarkably, this topological number is well-defined only when there are 
(a)

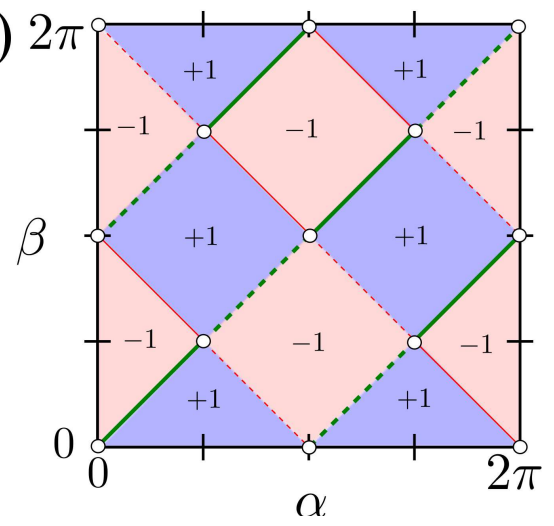

(b)

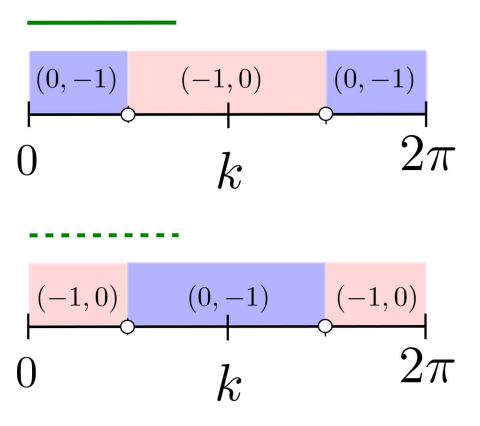

(c)

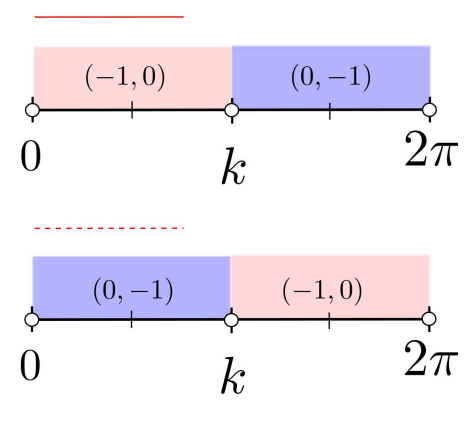

Figure 3: (a) The phase diagram of topological number $\nu_{2 d}$ derived in Ref. [4] as functions of $\alpha$ and $\beta$. In addition, the green thick and red thin (solid and dashed) lines represent vanishing gaps in the bulk spectrum

at the quasienergies of 0 and $\pi$, where the topological numbers $\left(\nu_{0}, \nu_{\pi}\right)$ are given in (4.27) and (4.31). (b and c) The wavenumber $k$ dependence of the topological numbers $\left(\nu_{0}, \nu_{\pi}\right)$ on the green thick and red thin (solid and dashed) lines, respectively. At the points shown by open circles in all figures, no topological numbers are defined. Note that the topological numbers on the red thin lines cannot be applied to the AE model.

no gaps in the two-dimensional bulk spectrum in order to retain chiral symmetry. This is the main result of this section, and we explain it hereafter.

First, we impose the following restrictions on $\alpha$ and $\beta$ in (4.17) to satisfy case (6) of Corollary 2 ,

$$
\beta \in\left\{\alpha+n \pi \mid n \in \mathbb{Z}, \alpha \in[0,2 \pi) \backslash\left\{0, \frac{\pi}{2}, \pi, \frac{3 \pi}{2}\right\}\right\} .
$$

Then, the time-evolution operator becomes

$$
\hat{\Gamma}_{0}\left(k_{x}, k_{y} ; n, \alpha\right)=(-1)^{n} \hat{\Gamma}_{0}\left(k_{x}, k_{y} ; \alpha\right), \quad \hat{\Gamma}_{0}\left(k_{x}, k_{y} ; \alpha\right)=\hat{D}\left(k_{x}\right) H_{\alpha} \hat{D}\left(k_{y}\right) H_{\alpha} .
$$

Since the prefactor $(-1)^{n}=e^{i n \pi}$ only shifts the quasi-energy by $n \pi$, we can focus on $\hat{\Gamma}_{0}\left(k_{x}, k_{y} ; \alpha\right)$ and recover the prefactor at the end of the calculations. Now, by shifting the origin of time to derive a time-evolution operator fitted in the symmetry time frame $[3]$, we obtain $\hat{\Gamma}_{0}^{\prime}\left(k_{x}, k_{y} ; \alpha\right)$

$$
\hat{\Gamma}_{0}^{\prime}\left(k_{x}, k_{y} ; \alpha\right)=\hat{D}\left(k_{x} / 2\right) H_{\alpha} \hat{D}\left(k_{y}\right) H_{\alpha} \hat{D}\left(k_{x} / 2\right) .
$$

We see that $\hat{\Gamma}_{0}^{\prime}\left(k_{x}, k_{y} ; \alpha\right)$ satisfies the relation

$$
\hat{Y} \hat{\Gamma}_{0}^{\prime}\left(k_{x}, k_{y} ; \alpha\right) \hat{Y}^{-1}=\hat{\Gamma}_{0}^{\prime}\left(k_{x}, k_{y} ; \alpha\right)^{-1},
$$

with chiral symmetry operator

$$
\hat{Y}=\sigma_{1} .
$$

Note that the presence of chiral symmetry relies on the condition (4.21) resulting in the absence of gaps in the bulk spectrum, as explained by Corollary $2(6)$. Since $\hat{\Gamma}_{0}^{\prime}\left(k_{x}, k_{y} ; \alpha\right)$ 
also retains particle-hole symmetry, the system with parameters (4.21) belongs to the class BDI. While the class BDI in two dimensions does not have finite topological numbers, chiral symmetry classes can have an integer topological number in one dimension 33]. Therefore, we fix $k_{y}$ of the time-evolution operator $\hat{\Gamma}_{0}^{\prime}\left(k_{x}, k_{y} ; \alpha\right)$ in (4.22) and regard it as a parameter, $k \in[0,2 \pi)$. In the end, we consider the following time-evolution operator,

$$
\hat{\Gamma}_{0}^{\prime}\left(k_{x} ; k, n\right)=(-1)^{n} \hat{\Gamma}_{0}^{\prime}\left(k_{x} ; k\right), \quad \hat{\Gamma}_{0}^{\prime}\left(k_{x} ; k\right)=\hat{D}\left(k_{x} / 2\right) H_{\alpha} \hat{D}(k) H_{\alpha} \hat{D}\left(k_{x} / 2\right) .
$$

Since $k$ is fixed, $\hat{\Gamma}_{0}^{\prime}\left(k_{x} ; k\right)$ effectively describes time-evolution in one dimension with chiral symmetry, while $\hat{D}(k)$ introduces a complex factor $e^{-i k \sigma_{3}}$. Therefore, the system belongs to the class AIII. Because of the presence of chiral symmetry, the topological number is calculated by the following the method in Ref. [3] as long as the gaps in the spectrum in the subspace $k_{x} \in[0,2 \pi)$ exist for a fixed $k$. In order to calculate the winding number, it is better to express $\hat{\Gamma}_{0}^{\prime}\left(k_{x} ; k\right)$ using Pauli matrices as

$$
\begin{aligned}
\hat{\Gamma}_{0}^{\prime}\left(k_{x} ; k\right) & =d_{0}^{\prime}\left(k_{x}\right) \sigma_{0}+i \sum_{j=1,2,3} d_{j}^{\prime}\left(k_{x}\right) \sigma_{j}, \\
d_{0}^{\prime}\left(k_{x}\right) & =\cos k \cos (2 \alpha) \cos k_{x}-\sin k \sin k_{x}, \\
d_{1}^{\prime}\left(k_{x}\right) & =0, \\
d_{2}^{\prime}\left(k_{x}\right) & =d_{2}^{\prime}=-\sin (2 \alpha) \cos k, \\
d_{3}^{\prime}\left(k_{x}\right) & =-\sin k \cos k_{x}-\cos k \cos (2 \alpha) \sin k_{x} .
\end{aligned}
$$

Furthermore, we apply a unitary transformation to $\hat{\Gamma}_{0}^{\prime}\left(k_{x} ; k\right)$ such that the chiral symmetry operator becomes $\hat{Y}=\sigma_{3}$,

$$
\begin{aligned}
\tilde{\Gamma}_{0}^{\prime}\left(k_{x} ; k\right) & =e^{-i \frac{\pi}{4} \sigma_{2}} \hat{\Gamma}_{0}^{\prime}\left(k_{x} ; k\right) e^{i \frac{\pi}{4} \sigma_{2}} \\
& =d_{0}^{\prime}\left(k_{x}\right) \sigma_{0}+i d_{3}^{\prime}\left(k_{x}\right) \sigma_{1}+i d_{2}^{\prime}\left(k_{x}\right) \sigma_{2} .
\end{aligned}
$$

The eigenvalue $\lambda_{ \pm}$and corresponding eigenvectors $\psi_{ \pm}^{\prime}\left(k_{x}\right)$ of $\tilde{\Gamma}_{0}^{\prime}\left(k_{x} ; k\right)$ are given by

$$
\begin{aligned}
\lambda_{ \pm} & =d_{0}^{\prime}\left(k_{x}\right) \pm i \sqrt{1-d_{0}^{\prime 2}\left(k_{x}\right)}, \\
\psi_{ \pm}^{\prime}\left(k_{x}\right) & =\frac{1}{\sqrt{2}}\left[\begin{array}{c}
\mp i e^{i \theta^{\prime}\left(k_{x}\right)} \\
1
\end{array}\right], \\
e^{i \theta^{\prime}\left(k_{x}\right)} & =\frac{d_{2}^{\prime}\left(k_{x}\right)+i d_{3}^{\prime}\left(k_{x}\right)}{\sqrt{1-d_{0}^{\prime 2}\left(k_{x}\right)}}
\end{aligned}
$$

Then, the winding number characterizing the topological properties of the eigenvector in wavenumber space is given by

$$
\begin{aligned}
\nu^{\prime} & =-\frac{i}{\pi} \int_{0}^{2 \pi} \overline{\psi_{ \pm}^{\prime}\left(k_{x}\right)} \frac{d \psi_{ \pm}^{\prime}\left(k_{x}\right)}{d k_{x}} d k_{x} \\
& =\frac{1}{2} \oint d \theta^{\prime}\left(k_{x}\right) .
\end{aligned}
$$

Therefore, the winding number is determined by whether $e^{i \theta^{\prime}\left(k_{x}\right)}$ in (4.25) forms a closed circle including the origin in the complex plane when $k_{x}$ changes from 0 to $2 \pi$. Since 
$\sqrt{1-d_{0}^{\prime 2}\left(k_{x}\right)}$ is positive finite as long as the spectrum $\lambda_{ \pm}$has gaps, we focus only upon the numerator in (4.25),$d_{2}^{\prime}\left(k_{x}\right)+i d_{3}^{\prime}\left(k_{x}\right)$, in the following argument. Then, because $d_{2}^{\prime}\left(k_{x}\right)$ is independent of $k_{x}, e^{i \theta^{\prime}\left(k_{x}\right)}$ does not wind around the origin and we have

$$
\nu^{\prime}=0
$$

for any $\alpha$ and $k$.

According to [3], in order to calculate the topological numbers $\nu_{0}$ and $\nu_{\pi}$ for quasienergies 0 and $\pi$, respectively, we need to calculate the winding number for the other time-evolution operator $\hat{\Gamma}_{0}^{\prime \prime}\left(k_{x} ; k\right)$ fitted in the different symmetry time frame

$$
\hat{\Gamma}_{0}^{\prime \prime}\left(k_{x} ; k\right)=\hat{D}(k / 2) H_{\alpha} \hat{D}\left(k_{x}\right) H_{\alpha} \hat{D}(k / 2) .
$$

Because of the relation $\hat{\Gamma}_{0}^{\prime \prime}\left(k_{x} ; k\right)=\hat{\Gamma}_{0}^{\prime}\left(k ; k_{x}\right)$, the winding number $\nu^{\prime \prime}$ of $\hat{\Gamma}_{0}^{\prime \prime}\left(k_{x} ; k\right)$ is given by switching $k_{x}$ and $k$ in the derivation of $\hat{\Gamma}_{0}^{\prime}\left(k ; k_{x}\right)$. In other words, the topological number $\nu^{\prime \prime}$ is given by

$$
\begin{aligned}
\nu^{\prime \prime} & =\frac{1}{2} \oint d \theta^{\prime \prime}\left(k_{x}\right), \\
e^{i \theta^{\prime \prime}\left(k_{x}\right)} & =\frac{d_{2}^{\prime \prime}\left(k_{x}\right)+i d_{3}^{\prime \prime}\left(k_{x}\right)}{\sqrt{1-d_{0}^{\prime \prime 2}\left(k_{x}\right)}} \\
d_{2}^{\prime \prime}\left(k_{x}\right) & =-\sin (2 \alpha) \cos k_{x}, \\
d_{3}^{\prime \prime}\left(k_{x}\right) & =-\cos k \sin k_{x}-\sin k \cos (2 \alpha) \cos k_{x} .
\end{aligned}
$$

Putting $u=d_{2}^{\prime \prime}\left(k_{x}\right)$ and $v=d_{3}^{\prime \prime}\left(k_{x}\right)$, we obtain the elliptic function of $u$ and $v$

$$
\left(\cos ^{2} k+\sin ^{2} k \cos ^{2}(2 \alpha)\right) u^{2}+\sin ^{2}(2 \alpha) v^{2}-2 \sin k \cos (2 \alpha) \sin (2 \alpha) u v=\cos ^{2} k \sin ^{2}(2 \alpha)
$$

By properly rotating the coordinate, the above elliptic function is simply written as

$$
\begin{aligned}
& u^{\prime 2}+\cos ^{2} k \sin ^{2}(2 \alpha) v^{\prime 2}=\cos ^{2} k \sin ^{2}(2 \alpha), \\
& {\left[\begin{array}{l}
u^{\prime} \\
v^{\prime}
\end{array}\right]=\left[\begin{array}{cr}
\cos \eta & \sin \eta \\
-\sin \eta & \cos \eta
\end{array}\right]\left[\begin{array}{l}
u \\
v
\end{array}\right],} \\
& \cos \eta=\frac{\cos (2 \alpha)}{\Xi}, \quad \sin \eta=\frac{\sin k \sin (2 \alpha)}{\Xi}, \quad \Xi=\sqrt{1-\cos ^{2} k \sin ^{2}(2 \alpha)} .
\end{aligned}
$$

From (4.26), the winding number $\nu^{\prime \prime}$ is determined as follows.

(1) The case of $\alpha=0, \frac{\pi}{2}, \pi, \frac{3 \pi}{2}$ : (these values are excluded by (4.21))

Since (4.26) is reduced to $u^{\prime}=u=0$, the trajectory of $\theta^{\prime \prime}\left(k_{x}\right)$ becomes a line, and then $\nu^{\prime \prime}=0$.

(2) The case of $k=\frac{\pi}{2}, \frac{3 \pi}{2}$ :

Since (4.26) is reduced to $u^{\prime}=\cos (2 \alpha) u \pm \sin (2 \alpha) v=0$, the trajectory of $\theta^{\prime \prime}\left(k_{x}\right)$ becomes a line and then $\nu^{\prime \prime}=0$. 
(3) Otherwise:

The trajectory of $\boldsymbol{p}=\left(u\left(k_{x}\right), v\left(k_{x}\right)\right) k_{x} \in[0,2 \pi)$ is a rotated ellipse enclosing the origin. The curvature of $\boldsymbol{p}$ is expressed by

$$
\kappa\left(k_{x}\right)=\frac{\dot{u}\left(k_{x}\right) \ddot{v}\left(k_{x}\right)-\ddot{u}\left(k_{x}\right) \dot{v}\left(k_{x}\right)}{\left(\dot{u}\left(k_{x}\right)^{2}+\dot{v}\left(k_{x}\right)^{2}\right)^{3 / 2}} .
$$

Here, $\dot{w}=d w / d k_{x}$, and $\ddot{w}=d^{2} w / d k_{x}^{2},(w=u, v)$. The numerator of $\kappa\left(k_{x}\right)$ in this case can be computed as $\sin 2 \alpha \cos k$. Thus, the sign of $\kappa$ coincides with that of $\sin 2 \alpha \cos k$, meaning $\nu^{\prime \prime} \neq 0$ if and only if $\sin 2 \alpha \cos k \neq 0$. Moreover, since $\dot{u}\left(k_{x}\right)=\sin k_{x} \sin 2 \alpha$, then $k_{x}=\pi$ is a unique point in $(0,2 \pi)$ such that the sign of $\dot{u}\left(k_{x}\right)$ is inverted, that is, for $\sin 2 \alpha>0$

$$
\dot{u}\left(k_{x}\right)>0\left(k_{x} \in(0, \pi)\right), \quad \dot{u}\left(k_{x}\right)<0\left(k_{x} \in(\pi, 2 \pi)\right),
$$

and vice versa for $\sin 2 \alpha<0$. Thus since the orbit is a rotated ellipse enclosing the origin, we have $\left|\nu^{\prime}\right|=1$. Taking into account the prefactor $(-1)^{n}$ in (4.24), we can conclude that

$$
\nu^{\prime \prime}= \begin{cases}1 & :(-1)^{n} \cos k \sin 2 \alpha>0, \\ -1 & :(-1)^{n} \cos k \sin 2 \alpha<0 .\end{cases}
$$

Finally, we use the formula to calculate the topological numbers for the zero and $\pi$ energy states, $\nu_{0}$ and $\nu_{\pi}$, respectively, for one-dimensional quantum walks with chiral symmetry[3]:

$$
\left(\nu_{0}, \nu_{\pi}\right)=\left(\frac{\nu^{\prime}+\nu^{\prime \prime}-1}{2}, \frac{\nu^{\prime}-\nu^{\prime \prime}-1}{2}\right) .
$$

Putting $\nu^{\prime}$ and $\nu^{\prime \prime}$ into the above formula, we have

$$
\left(\nu_{0}, \nu_{\pi}\right)= \begin{cases}(0,-1) & :(-1)^{n} \cos k \sin 2 \alpha>0 \\ (-1,0) & :(-1)^{n} \cos k \sin 2 \alpha<0 \\ \text { undefined } & : \cos k \sin 2 \alpha=0\end{cases}
$$

The above result is summarized in figures 3 (a) and (b). We remark that topological numbers $\nu_{0}$ and $\nu_{\pi}$ depend not only upon the angle of the coin operators $\alpha$ and $n$ but also the wavenumber $k$. Since the absolute values of both topological numbers is one, we predict single edge states at quasi-energies of 0 and $\pi$ for each $k$ using the bulk-edge correspondence. Therefore, in the semi-infinite space $V$ where $k$ is continuous in $[0,2 \pi)$, the number of edge states at quasi-energies 0 and $\pi$ become infinite as a consequence of the accumulation of topological numbers for each $k$. Then, infinitely degenerated edge states develop wavenumber independent dispersion relations at quasi-energies 0 and $\pi$, which is consistent with the proof of the case (6) in Corollary 2.

The above procedure can also be applied to case (1) in Corollary 2 ,

$$
\beta \in\{-\alpha+n \pi \mid n \in \mathbb{Z}, \alpha \in[0,2 \pi)\},
$$

where there are neither gaps in the bulk spectrum, nor and there are no edge states. Putting (4.28) into (4.17) and regarding $k_{y}$ as a fixed parameter $k \in[0,2 \pi)$, the time-evolution 
operator in the effective one-dimensional fitted in symmetry time frame is expressed as

$$
\begin{aligned}
\check{Y} \check{\Gamma}_{0}^{\prime}\left(k_{x} ; k\right) \check{Y}^{-1} & =\check{\Gamma}_{0}^{\prime}\left(k_{x} ; k\right)^{-1}, \\
\check{\Gamma}_{0}^{\prime}\left(k_{x} ; k\right) & =\hat{D}\left(k_{x} / 2\right) H_{\alpha} \hat{D}(k) H_{-\alpha} \hat{D}\left(k_{x} / 2\right), \\
\check{\Gamma}_{0}^{\prime}\left(k_{x} ; k, n\right) & =(-1)^{n} \Gamma_{0}^{\prime}\left(k_{x} ; k\right)
\end{aligned}
$$

with the chiral symmetry operator

$$
\check{Y}=\sigma_{2}
$$

More explicitly, $\check{\Gamma}_{0}^{\prime}\left(k_{x} ; k\right)$ is written as

$$
\begin{aligned}
\check{\Gamma}_{0}^{\prime}\left(k_{x} ; k\right) & =e_{0}^{\prime}\left(k_{x}\right) \sigma_{0}+i \sum_{j=1,2,3} e_{j}^{\prime}\left(k_{x}\right) \sigma_{j}, \\
e_{0}^{\prime}\left(k_{x}\right) & =\cos k \cos k_{x}-\sin k \cos (2 \alpha) \sin k_{x}, \\
e_{1}^{\prime}\left(k_{x}\right) & =-\sin k \sin (2 \alpha), \\
e_{2}^{\prime}\left(k_{x}\right) & =0, \\
e_{3}^{\prime}\left(k_{x}\right) & =-\cos k \sin k_{x}-\sin k \cos (2 \alpha) \cos k_{x} .
\end{aligned}
$$

We remark that the chiral symmetry operators $\check{Y}$ is different from $\hat{Y}$ in (4.23). Applying the unitary transform $e^{i \frac{\pi}{4} \sigma_{1}} \check{\Gamma}_{0}^{\prime}\left(k_{x} ; k\right) e^{-i \frac{\pi}{4} \sigma_{1}}$, the topological numbers for energies of zero and $\pi$, $\nu_{0}$ and $\nu_{\pi}$, are derived through the same procedure as before. The result is summarized as

$$
\left(\nu_{0}, \nu_{\pi}\right)= \begin{cases}(-1,0) & :(-1)^{n} \sin k \sin 2 \alpha>0 \\ (0,-1) & :(-1)^{n} \sin k \sin 2 \alpha<0 \\ \text { undefined } & : \sin k \sin 2 \alpha=0\end{cases}
$$

We summarize the above result in figures 3 (a) and (c). We note, however, that the topological numbers in (4.31) cannot be applied to the AE model for the reason explained below.

Remarkably, when we consider case (1) in Corollary 2, which proves the absence of edge states, topological numbers $\nu_{0}$ and $\nu_{\pi}$ become finite, except $\sin k \sin 2 \alpha=0$. However, when we predict the number of edge states using the bulk-edge correspondence for the system with a boundary, we keep in mind that the boundary does not break the symmetry retained in the bulk. Then, we check whether the boundary realized by the shift operator in (A.1) [or (A.3)] retains chiral symmetry. Since we consider the shift operator in the position space in (A.1), we redefine the chiral symmetry operators as

$$
\hat{Y}=\sum_{x, y}|x, y\rangle\left\langle x, y\left|\otimes\left[\begin{array}{cc}
\sigma_{1} & 0 \\
0 & \sigma_{1}
\end{array}\right], \quad \check{Y}=\sum_{x, y}\right| x, y\right\rangle\langle x, y| \otimes\left[\begin{array}{cc}
\sigma_{2} & 0 \\
0 & \sigma_{2}
\end{array}\right] .
$$

In order to retain chiral symmetry, the relation $X S^{\prime \prime} X^{-1}=S^{\prime \prime-1}(X \in\{\hat{Y}, \check{Y}\})$ should be satisfied. We can confirm that

$$
\begin{aligned}
\hat{Y} S^{\prime \prime} \hat{Y} S^{\prime \prime} & =\sum_{x, y}|x, y\rangle\langle x, y| \otimes(|R\rangle\langle R|+| L\rangle\langle L|+| D\rangle\langle D|+| U\rangle\langle U|) \\
& =\sum_{x, y}|x, y\rangle\langle x, y| \otimes I_{4} \quad:(x, y) \in V
\end{aligned}
$$


where $I_{4}$ is an identity matrix of $4 \times 4$ matrices, while

$$
\check{Y} S^{\prime \prime} \check{Y} S^{\prime \prime}= \begin{cases}\sum_{x, y}|x, y\rangle\langle x, y| \otimes I_{4} & :(x, y) \notin \partial V, \\ \sum_{x, y}|x, y\rangle\langle x, y| \otimes(-|R\rangle\langle R|+| L\rangle\langle L|+| D\rangle\langle D|+| U\rangle\langle U|) & :(x, y) \in \partial V .\end{cases}
$$

Therefore, the boundary retains chiral symmetry given by $\hat{Y}$ in (4.23), while it breaks chiral symmetry defined by the symmetry operator $\check{Y}$ in (4.30). Thus, the topological numbers in (4.31) cannot be applied to the AE model.

We note that a boundary realized by a shift operator with an extra phase $i$ on the self loop

$$
\begin{aligned}
& S_{i}^{\prime \prime}|x, y\rangle|J\rangle=S^{\prime}|x, y\rangle|J\rangle,(J \in\{R, D, U\}), \\
& S_{i}^{\prime \prime}|x, y\rangle|L\rangle= \begin{cases}S^{\prime}|x, y\rangle|L\rangle & :(x, y) \notin \partial V, \\
i S^{\prime}|x, y\rangle|L\rangle & :(x, y) \in \partial V,\end{cases}
\end{aligned}
$$

satisfies $\check{Y} S_{i}^{\prime \prime} \check{Y}^{-1}=S_{i}^{\prime \prime-1}$, but $\hat{Y} S_{i}^{\prime \prime} \hat{Y}^{-1} \neq S_{i}^{\prime \prime-1}$. With the shift operator $S_{i}^{\prime \prime}$, we numerically confirm that a wavenumber independent dispersion relation for the edge states appears and agrees with the topological numbers in (4.31) for the parameters in case (1) in Corollary 2, whereas there appear to be no edge states in case (6).

\subsection{Demonstrations}

Here, we present plots of dispersion relations for bulk and edge states, which are written in Theorem 2, for specific values of $\alpha$ and $\beta$ in Figures 4,5 and compare them with predictions based on topological numbers. According to the bulk-edge correspondence, if topological numbers $\nu_{2 d}, \nu_{0}$, and $\nu_{\pi}$ are finite, we expect edge states. We can confirm that the dispersion relations for edge states agree well with the prediction based on topological numbers. For the triple of the signs $\left(\epsilon_{1}, \epsilon_{2}, \epsilon_{3}\right),\left(\epsilon_{j} \in\{ \pm\}\right)$, where $\epsilon_{1}=\operatorname{sgn}(\sin 2 \alpha \sin 2 \beta), \epsilon_{2}=\operatorname{sgn}(\sin (\alpha+\beta)$, and $\epsilon_{3}=\operatorname{sgn}(\sin (\alpha-\beta))$, we have the topological number $\nu_{2 d}=\epsilon_{2} \epsilon_{3}$.

\section{$5 \quad$ Asymptotic behavior of the quantum walk}

We have obtained the dispersion relation of the quantum walk, and found the existence of the edge spectrum in gaps of the bulk spectrum, which agrees with the prediction by the bulk-edge corresponding of topological numbers. Moreover, in the next section, we shown that the states corresponding to the edge spectrum exponentially localize near self-loops on the boundary. These observations give a certification to call these states the edge states. In this section, we calculate the finding probability along the boundary. In the previous studies on the quantum walk on the one dimensional lattice related to the topological phases, the response on the finding probability is characterized by a specific property of quantum walks named the localization [20, 29]. Since our model treats two-dimensional model, our interest is naturally how the response is deformed in this higher dimensional system.

To this end, we concentrate upon the finding probability at the boundaries $\partial V$ to observe how the quantum walker recognizes the boundary: we set $\nu_{n}: \mathbb{Z} \rightarrow[0,1]$ as the probability 


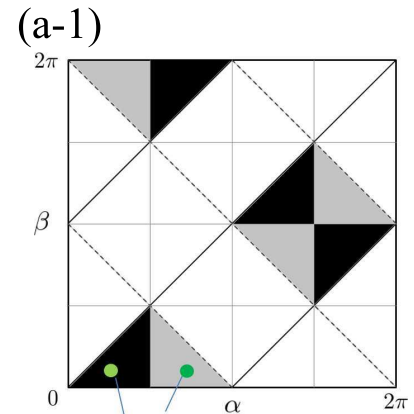

(A) $(B)$

(b-1)

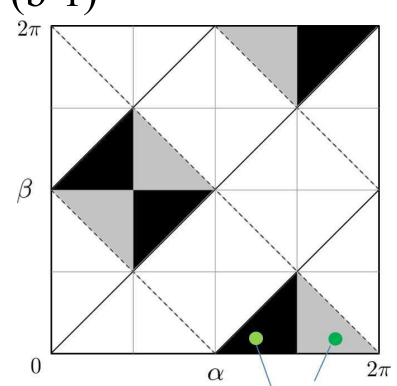

(C) (D)

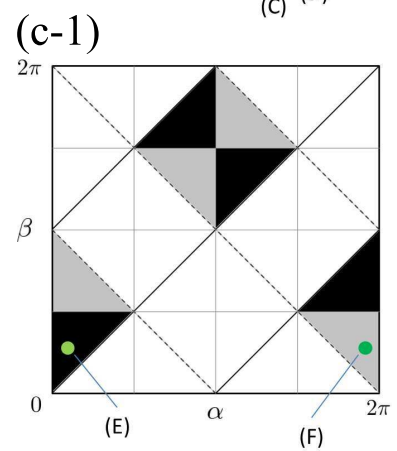

(d-1)

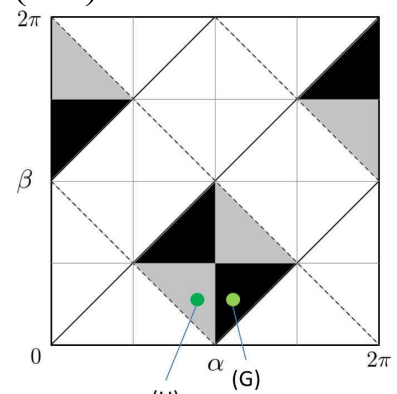

(a-2)

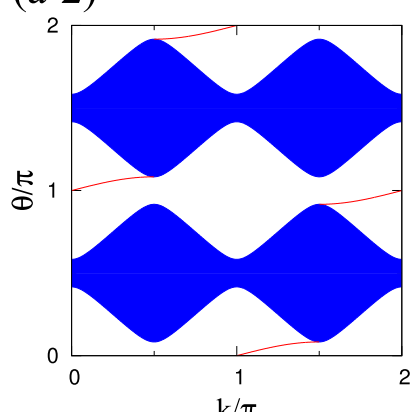

(b-2)

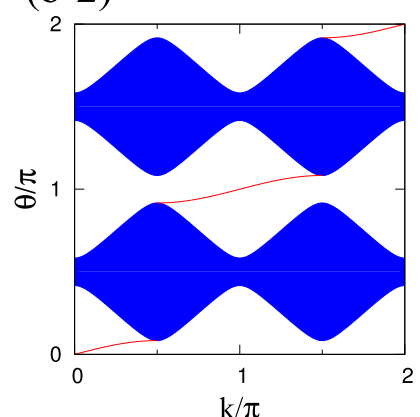

(c-2)

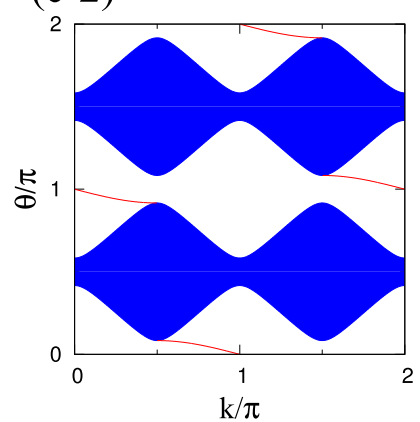

(d-2)

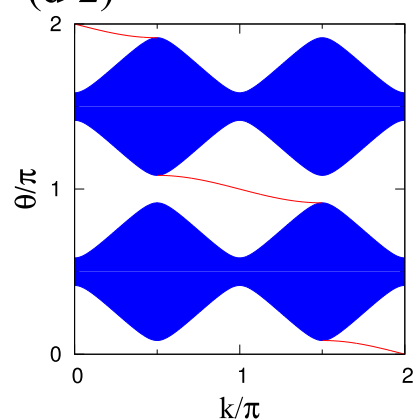

(a-3)

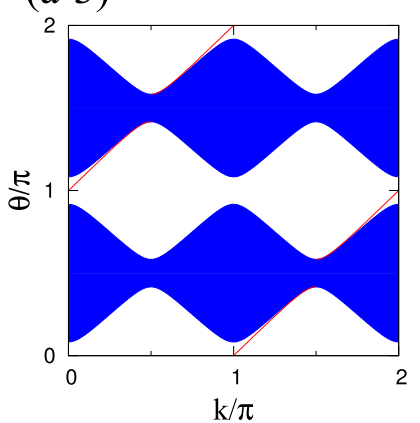

(b-3)

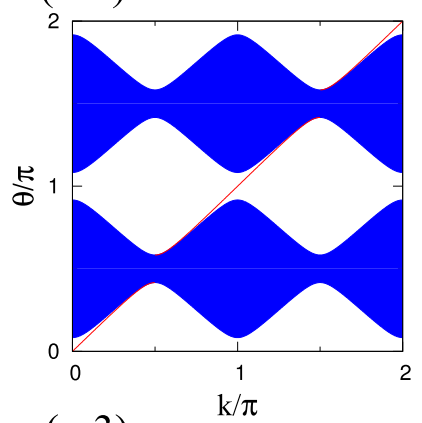

(c-3)

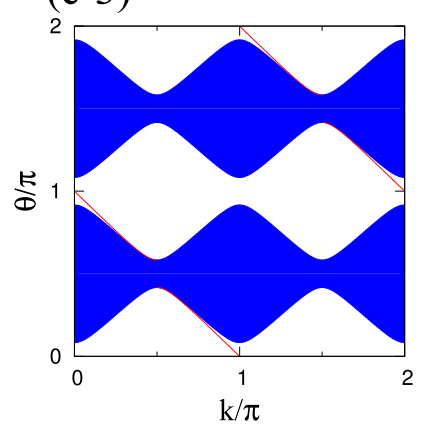

(d-3)

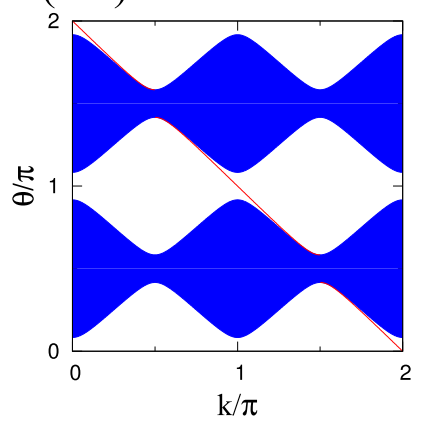

Figure 4: (left column) The black and gray regions represents two parameter classes $\left(\varepsilon_{1}, \varepsilon_{2}, \varepsilon_{3}\right)$ considered the middle and right columns, respectively. (middle and right columns) The dispersion relations for bulk (filled blue regions) and edge states (red curves) for a specific value of $(\alpha, \beta)$ included in two parameter classes shown in the left column. The specific values of $(\alpha, \beta)$ are as follows: $(\mathrm{a}-2)(\mathrm{A}):(\pi / 4, \pi / 6) \in(+,+,+),(\mathrm{a}-3)(\mathrm{B}):(3 \pi / 4, \pi / 6) \in(-,+,+)$, (b-2) $(\mathrm{C}):(5 \pi / 4, \pi / 6) \in(+,-,-),(\mathrm{b}-3)(\mathrm{D}):(7 \pi / 4, \pi / 6) \in(-,-,-),(\mathrm{c}-2)(\mathrm{E}):(\pi / 6, \pi / 4) \in$ $(+,+,-),(\mathrm{c}-3)(\mathrm{F}):(11 \pi / 6, \pi / 4) \in(-,+,-),(\mathrm{d}-2)(\mathrm{G}):(7 \pi / 6, \pi / 4) \in(+,-,+)$, and $(\mathrm{d}-$ 3) $(\mathrm{H}):(5 \pi / 6, \pi / 4) \in(-,-,+)$. The topological number is $\nu_{2 d}=+1$ in (a) and (b), but $\nu_{2 d}=-1$ in $(\mathrm{c})$ and $(\mathrm{d})$. 
$(\mathrm{a}-1)$

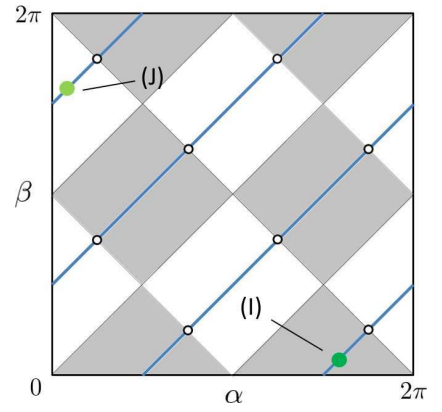

(b-1)

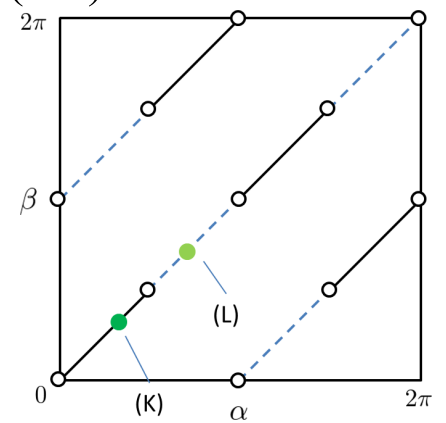

$(\mathrm{c}-1)$

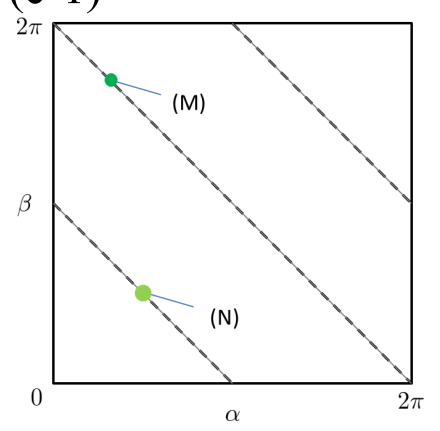

$(\mathrm{a}-2)$

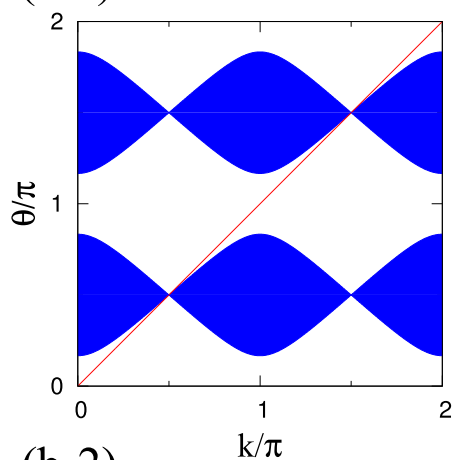

(b-2)

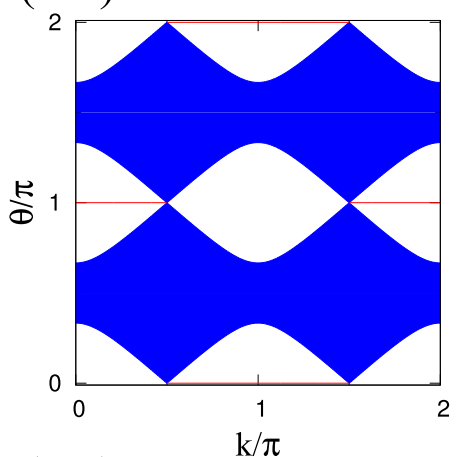

(c-2)

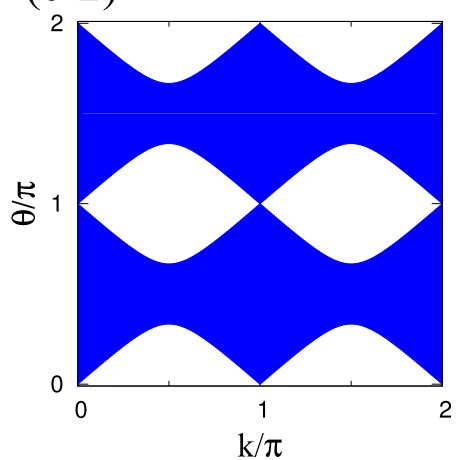

(a-3)

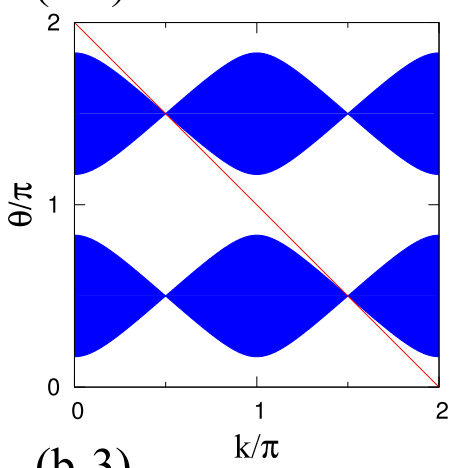

(b-3)

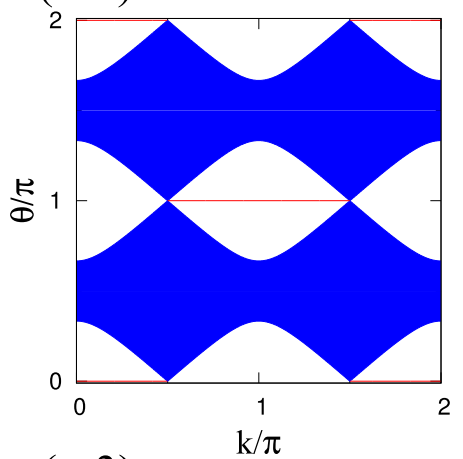

$(\mathrm{c}-3)$

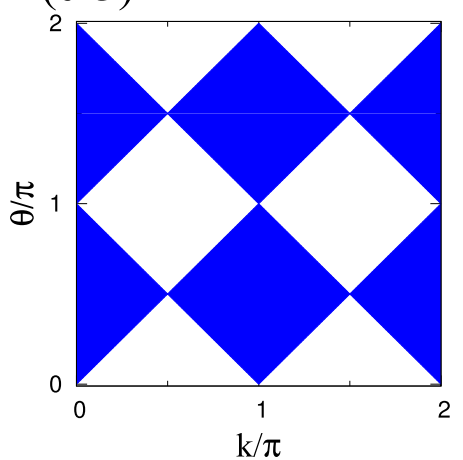

Figure 5: (left column) The black and gray regions represents two parameter classes $\left(\varepsilon_{1}, \varepsilon_{2}, \varepsilon_{3}\right)$ considered the middle and right columns, respectively. (middle and right columns) The dispersion relations for bulk (filled blue regions) and edge states (red curves) for a specific value of $(\alpha, \beta)$ included in two parameter classes shown in the left column. The specific values of $(\alpha, \beta)$ are as follows: (a-2) (I): $(5 \pi / 3, \pi / 6) \in(-,-,-),(\mathrm{a}-3)(\mathrm{J}):(\pi / 6,5 \pi / 3) \in(-,-,+)$, $(\mathrm{b}-2)(\mathrm{K}):(\pi / 3, \pi / 3) \in(+,+, 0),(\mathrm{b}-3)(\mathrm{L}):(2 \pi / 3,2 \pi / 3) \in(+,-, 0)(\mathrm{c}-2)(\mathrm{M}):(\pi / 3,5 \pi / 3) \in$ $(-, 0,+)$, and $(\mathrm{c}-3)(\mathrm{N}):(\pi / 4,3 \pi / 4) \in(-, 0,-)$ In $(\mathrm{a})$, the topological number is $\nu_{2 d}=+1$. In (b-1), the real lines depict the parameter classes of $(+,+, 0)$ where the topological numbers are given in figure $2(\mathrm{~b})$. The dashed lines depict $(+,-, 0)$ where the topological numbers are given in figure2(c). In (c), there are no well-defined topological numbers. 
that a quantum walker is measured at time $n$ at the boundary,

$$
\nu_{n}(j)=\left|\left(U^{2 n} \psi_{0}\right)\left(a_{j}\right)\right|^{2} .
$$

Here $a_{j} \in A$ is the self-loop at $[0, j] \in V$. Recall that the initial state is fixed to the self-loop at the origin $a_{0} \in A$. We have

$$
\hat{\psi}_{n}(k):=\sum_{j \in \mathbb{Z}}\left(U^{2 n} \psi_{0}\right)\left(a_{j}\right) e^{i k j}=\hat{\varphi}_{n, 1}(0 ; k) .
$$

Now we define some asymptotic behaviors of $\nu_{n}$ which will be the responses to the cutting edges of the quantum walk as follows.

\section{Definition 6.}

(1) We say linear spreading occurs at the boundary if and only if there exists a monotonically non-decreasing function $F(\neq 0): \mathbb{R}_{\geq 0} \rightarrow[0,1]$, which is not given by $c_{0} \mathrm{H}(\cdot)$ with some non-zero constant $c_{0}$, such that

$$
\lim _{n \rightarrow \infty} \sum_{|j|<n y} \nu_{n}(j)=F(y) \text { for any } y \in \mathbb{R}_{\geq 0} .
$$

Here $\mathrm{H}(x)$ is the unit step function, that is,

$$
\mathrm{H}(x)= \begin{cases}1 & : x \geq 0 \\ 0 & : x<0\end{cases}
$$

In particular,

(a) if $F(y)$ is continuous on $\mathbb{R}_{\geq 0}$, then we say continuously-linear spreading occurs;

(b) if $F(y)$ is described by $c_{0} \mathrm{H}(y-1)$, then we say ballistic spreading occurs.

(2) We say localization occurs at the boundary if and only if there exists $j \in \mathbb{Z}$ such that

$$
\limsup _{T \rightarrow \infty} \frac{1}{T} \sum_{n=0}^{T-1} \nu_{n}(j)>0 .
$$

Remark 4. If there exists $q \in[0,1)$ and monotonic function $G(\neq 0): \mathbb{R}_{\geq 0} \rightarrow[0,1]$ such that

$$
\lim _{n \rightarrow \infty} \sum_{|j|<n^{q} y} \nu_{n}(j)=G(y)
$$

then

$$
\lim _{n \rightarrow \infty} \sum_{|j|<n y} \nu_{n}(j)=\lim _{n \rightarrow \infty} G\left(n^{1-q} y\right)=\lim _{n \rightarrow \infty} c_{0} \mathrm{H}(y)
$$

holds, where $c_{0}=\lim _{y \rightarrow \infty} G(y)$. Thus the condition that $F \neq c_{0} \mathrm{H}$ for the definition of the linear spreading prohibits such a slow spreading. 
We have the following theorem:

Theorem 3. Let us define $s:=\sin (\alpha+\beta) \neq 0$, and $r:=\sin (\alpha-\beta)$.

(1) If $0<|r|<1$, then the continuously linear spreading occurs: for any $a<b \in \mathbb{R}$,

$$
\lim _{n \rightarrow \infty} \sum_{a \leq j / n \leq b} \nu_{n}(j)=\int_{a}^{b} g(y) d y
$$

Here the density of $F(y)$ is

$$
g(y)=\frac{2 s^{2}}{r^{2}} y^{2} f_{K}(y ;|r|) \times \begin{cases}\mathbf{1}_{[0, \infty)}(y) & : s r>0 \\ \mathbf{1}_{(-\infty, 0]}(y) & : s r<0\end{cases}
$$

where $f_{K}(x ;|r|)$ is the Konno function with the parameter $|r|$.

(2) If $|r|=1$, then the ballistic spreading occurs:

$$
\lim _{n \rightarrow \infty} \sum_{-\infty \leq j / n \leq y} \nu_{n}(j)=s^{2} \mathrm{H}(y-\operatorname{sgn}(r s))
$$

which implies the density of $F(y)$ is $s^{2} \delta(y-\operatorname{sgn}(r s))$. Moreover it holds that

$$
\lim _{n \rightarrow \infty} \nu_{n}(\operatorname{sgn}(r s)(n-j))=s^{2} \delta_{0}(j) .
$$

(3) If $|r|=0$, then localization occurs:

$$
\begin{gathered}
\lim _{n \rightarrow \infty} \nu_{2 n}(j)= \begin{cases}\frac{s^{2}}{\pi^{2}} \frac{4}{\left(j^{2}-1\right)^{2}} & : j \text { is even }, \\
0 & : \text { otherwise. }\end{cases} \\
\lim _{n \rightarrow \infty} \nu_{2 n+1}(j)= \begin{cases}\frac{s^{2}}{4} & : j \in\{ \pm 1\} \\
0 & : \text { otherwise. }\end{cases}
\end{gathered}
$$

Here $f_{K}$ is the density of the Konno distribution [23, 24] with the parameter $p \in(0,1)$ denoted by

$$
f_{K}(x ; p)=\frac{\sqrt{1-p^{2}}}{\pi\left(1-x^{2}\right) \sqrt{p^{2}-x^{2}}} \mathbf{1}_{(-p, p)}(x) .
$$

The dispersion relation classes of the limit theorem in the parameter setting of (3) in Theorem 3, that is, $|r|=|\sin (\alpha-\beta)|=0$, is $\left(\epsilon_{1}, \epsilon_{2}, 0\right),\left(\epsilon_{j} \in\{ \pm\}(j=1,2)\right)$. In this case, we have a wave number independent dispersion relation, that is, $\theta_{0}(k) / d k=0$ for all $k$. On the other hand, the dispersion relation classes of the limit theorem in the parameter setting of (2), that is, $|r|=|\sin (\alpha-\beta)|=1$, is a special class of $\left(-, \epsilon_{2}, \epsilon_{3}\right),\left(\epsilon_{j} \in\{ \pm\}(j=2,3)\right)$. In this case, we have the linear dispersion relation for the edge state, that is, $\left|\theta_{0}(k) / d k\right|=1$ for all $k$. The the wave number independence and linearity of the edge state are reflected in the stochastic behaviors of the quantum walk on the boundary as localization and ballistic spreading, respectively. 
Proof. The $n$-th iteration of the CMV matrix is expressed by a "quantum" version of the formula corresponding to the Karlin MacGregor formula for random walks:

$$
\left(\mathcal{C}^{n}\right)_{i, j}=\int_{|z|=1} z^{n} \overline{\chi_{i}(z)} \chi_{j}(z) d \mu(z)
$$

Concerning the one-to-one correspondence between the canonical bases of $\mathcal{C}$ and the Type-I quantum walk described by Lemma 4, $\mathcal{C}_{0,0}^{n}$ expresses the complex-valued amplitude at return to the origin at time $n$, that is,

$$
\mathcal{C}_{0,0}^{n}=\hat{\psi}_{n}(k) \text {. }
$$

Combining (5.9) with (5.10) and Lemma 6, we have

$$
\hat{\psi}_{n}(k)=\int_{0}^{2 \pi} e^{i n \theta}\left(\frac{w(\theta)}{2 \pi}+m_{0}(k) \delta\left(\theta_{0}(k)-\theta\right)\right) d \theta .
$$

The Riemann and Lebesgue lemma implies

$$
\hat{\psi}_{n}(k)=e^{i n \theta_{0}(k)} m_{0}(k)+o(1 / \sqrt{n}) .
$$

The assumption that $s \neq 0$ ensures $m_{0}(k) \neq 0$ for almost every $k$. We define the Fourier transform of $\nu_{n}$ by $\phi_{n}(\xi):=\sum_{j \in \mathbb{Z}} \nu_{n}(j) e^{i \xi j}(\xi \in \mathbb{R})$. Here explicit expression of $\theta_{0}(k)$ and $m_{0}(k)$ are described by (4.2) and (4.3), respectively. Now we will prove our statement for each case.

Proof of part (1). It holds that

$$
\phi_{n}(\xi)=\int_{0}^{2 \pi} \overline{\hat{\psi}_{n}(k)} \hat{\psi}_{n}(k+\xi) \frac{d k}{2 \pi} .
$$

Under the assumption $\sin (\alpha-\beta) \sin (\alpha+\beta) \neq 0$, then using the expression (5.12), we obtain

$$
\phi_{n}(\xi / n)=\int_{0}^{2 \pi} e^{i \xi v(k)} m_{0}^{2}(k) \frac{d k}{2 \pi}+o(1 / \sqrt{n})
$$

where $v(k)=d \theta_{0}(k) / d k$ :

$$
\frac{d \theta_{0}}{d k}=\operatorname{sgn}(\sin (\alpha-\beta) \sin (\alpha+\beta))|\sin (\alpha-\beta)| \frac{|\cos k|}{\sqrt{1-\sin ^{2}(\alpha-\beta)} \sin ^{2} k} .
$$

Since

$$
\frac{d}{d k}\left(\frac{\cos k}{\sqrt{1-r^{2} \sin ^{2} k}}\right)=-\frac{\sin k \cos ^{2}(\alpha-\beta)}{\left(1-\sin ^{2}(\alpha-\beta) \sin ^{2} k\right)^{3 / 2}},
$$

we have $0 \leq v(k) \leq|r|(r s>0)$ and $-|r| \leq v(k) \leq 0(r s<0)$. We consider $r s>0$ case. For $0 \leq y \leq|r|$, due to the assumption $|r|<1$, the solutions for $y=v(k)$ with respect to $k$ are $k_{1}<k_{2}<k_{3}<k_{4}$, which satisfy

$$
\sin ^{2} k_{j}=\frac{r^{2}-y^{2}}{r^{2}\left(1-y^{2}\right)} \quad(j=1,2,3,4)
$$


which implies $0<k_{1}<\pi / 2<k_{2}<\pi<k_{3}<3 \pi / 2<2 \pi$, and $k(y):=k_{1}, k_{2}=\pi-k(y)$, $k_{3}=\pi+k(y)$ and $k_{4}=2 \pi-k(y)$. We divide the integration (5.14) into the four parts: $\int_{0}^{\pi / 2}+\int_{\pi / 2}^{\pi}+\int_{\pi}^{3 \pi / 2}+\int_{3 \pi / 2}^{2 \pi}$. By taking $x=v(k)$ together with (5.16), we have

$$
\frac{d v(k)}{d k}=-\frac{\left(1-y^{2}\right) \sqrt{r^{2}-y^{2}}}{\sqrt{1-r^{2}}}=\frac{1}{\pi f_{K}(y ; r)} .
$$

and

$$
m_{0}^{2}(k)=\frac{s^{2}}{r^{2}} y^{2}
$$

Then it holds

$$
\begin{aligned}
I_{1}(\xi) & :=\int_{0}^{\pi / 2} e^{i \xi v(k)} m_{0}^{2}(k) \frac{d k}{2 \pi} \\
& =\int_{0}^{|r|} e^{i \xi y} \frac{s^{2}}{r^{2}} y^{2} \frac{1}{2} f_{K}(y ; r) d y .
\end{aligned}
$$

In a similar fashion, we have $I_{1}(\xi)=I_{2}(\xi)=I_{3}(\xi)=I_{4}(\xi)$. Therefore we can rewrite (5.14) by

$$
\phi(\xi):=\lim _{n \rightarrow \infty} \phi(\xi / n)=\int_{0}^{|r|} e^{i \xi y} \frac{s^{2}}{r^{2}} y^{2} f_{K}(y ; r) d y .
$$

In the same way, for $r s<0$ case we have

$$
\lim _{n \rightarrow \infty} \phi(\xi / n)=\int_{-|r|}^{0} e^{i \xi y} \frac{s^{2}}{r^{2}} y^{2} f_{K}(y ; r) d y
$$

Since $\phi(\xi)$ is continuous at $\xi=0$, then the continuity theorem implies that, for any $a<b \in \mathbb{R}$,

$$
\lim _{n \rightarrow \infty} \sum_{a \leq j / n \leq b} \nu_{n}(j)=\int_{a}^{b} g(y) d y
$$

Proof of part (2) . Since $|r|=1$, then $m_{0}(k)$ and $\theta_{0}(k)$ are reduced to $m_{0}(k)=|s|$, and

$$
\theta_{0}(k)= \begin{cases}\pi+k & :(\operatorname{sgn}(s), \operatorname{sgn}(r))=(+,+), \\ \pi-k & :(\operatorname{sgn}(s), \operatorname{sgn}(r))=(+,-), \\ 2 \pi-k & :(\operatorname{sgn}(s), \operatorname{sgn}(r))=(+,-), \\ k & :(\operatorname{sgn}(s), \operatorname{sgn}(r))=(-,-),\end{cases}
$$

respectively. From (5.12) and (5.13), the Fourier transform of $\nu_{n}$ is expressed by

$$
\phi_{n}(\xi / n) \sim s^{2} \begin{cases}e^{i \xi} & :(\operatorname{sgn}(s), \operatorname{sgn}(r)) \in\{(+,+),(-,-)\} \\ e^{i \xi} & :(\operatorname{sgn}(s), \operatorname{sgn}(r)) \in\{(+,-),(-,+)\} .\end{cases}
$$


Therefore $F(y)=s^{2} \mathbf{1}_{[1, \infty)}(y)$ holds. We set $\tau(j):=\left(U^{2 n} \psi_{0}\right)\left(a_{j}\right)$. Taking the inverse Fourier transform with insertion of these values into $m_{0}(k)$ and $\theta_{0}(k)$ in (5.12), we have

$$
\begin{aligned}
\tau(n-j) & :=\left(U^{2 n} \psi\right)\left(a_{n-j}\right) \sim \int_{0}^{2 \pi} e^{i n \theta_{0}(k)} m_{0}(k) e^{-i(n-j) k} \frac{d k}{2 \pi} \\
& =(-1)^{n}|s| \delta_{0}(j)
\end{aligned}
$$

for the $(\operatorname{sgn}(s), \operatorname{sgn}(r))=(+,+)$ and $(-,-)$ cases. In the same way, we have the $\tau(-n+j) \sim$ $(-1)^{n}|s| \delta_{0}(j)(\operatorname{sgn}(s), \operatorname{sgn}(r))=(+,-)$ and $(-,+)$ cases.

Proof of part (3). Let $B_{1}=(-\pi / 2, \pi / 2) \bmod (2 \pi)$, and $B_{2}=(\pi / 2,3 \pi / 2)$. Since $r=0$, $\theta_{0}(k)$ is flat, that is, by (4.3),

$$
\theta_{0}(k)= \begin{cases}\pi & : k \in B_{1} \\ 0 & : k \in B_{2}\end{cases}
$$

for $s>0$, and

$$
\theta_{0}(k)= \begin{cases}0 & : k \in B_{1} \\ \pi & : k \in B_{2}\end{cases}
$$

for $s<0$. Moreover, $m_{0}(k)$ is reduced to $|s||\cos k|$. Then we have

$$
\begin{aligned}
& \hat{\psi}_{n}(k)=|s| \times\left\{\begin{array}{ll}
(-1)^{n}|\cos k| & : k \in B_{1} \\
|\cos k| & : k \in B_{2}
\end{array} \quad(s>0)\right. \\
& \hat{\psi}_{n}(k)=|s| \times\left\{\begin{array}{ll}
|\cos k| & : k \in B_{1} \\
(-1)^{n}|\cos k| & : k \in B_{2}
\end{array} \quad(s<0)\right.
\end{aligned}
$$

For $s>0$, taking the inverse Fourier transform to $\hat{\psi}_{n}(k)$, we have

$$
\begin{aligned}
\tau_{n}(j) & \sim \int_{0}^{2 \pi} \hat{\psi}_{n}(k) e^{-i k j} \frac{d k}{2 \pi} \\
& =|s|\left\{(-1)^{n} \int_{k \in B_{1}} \cos k e^{-i k j} \frac{d k}{2 \pi}+\int_{k \in B_{2}}|\cos k| e^{-i k j} \frac{d k}{2 \pi}\right\}
\end{aligned}
$$

We have

$$
\begin{aligned}
& J_{1}(j):=\int_{k \in B_{1}} \cos k e^{-i k j} \frac{d k}{2 \pi}= \begin{cases}\frac{(-1)^{j / 2}}{\pi} \frac{1}{1-j^{2}} & : j \text { is even } \\
1 / 4 & : j \in\{ \pm 1\} \\
0 & : \text { otherwise }\end{cases} \\
& J_{2}(j):=\int_{k \in B_{2}}|\cos k| e^{-i k j} \frac{d k}{2 \pi}= \begin{cases}\frac{(-1)^{j / 2}}{\pi} \frac{1}{1-j^{2}} & : j \text { is even, } \\
-1 / 4 & : j \in\{ \pm 1\} \\
0 & : \text { otherwise. }\end{cases}
\end{aligned}
$$

Then it holds that

$$
\tau_{n}(j) \sim|s|\left((-1)^{n} J_{1}(j)+J_{2}(j)\right)= \begin{cases}\frac{1+(-1)^{n}}{2} 2|s|(-1)^{j / 2} /\left(\pi\left(1-j^{2}\right)\right) & : j \text { is even } \\ \frac{1+(-1)^{n-1}}{2}|s| / 2 & : j \in\{ \pm 1\} \\ 0 & : \text { otherwise }\end{cases}
$$


In the same way for $s<0$, we obtain the same expression for $\tau_{n}(j)$. Taking the square modulus to $\tau_{n}(j)$, we obtain the desired conclusion.

The group-velocity of the edge state is $v(k)=d \theta_{0}(k) / d k$, and the effective mass of the edge state is $M(k)=\left|1 /\left(d^{2} \theta_{0}^{2}(k) / d k^{2}\right)\right|$. The limit distribution is expressed by the above physical quantities:

Corollary 3. Let $v(k), M(k)$ be the above. The density function of the limit distribution for $0<|\sin (\alpha-\beta)|<1$ is denoted by $g(\cdot)$. Then we have the following parametric plot of the density function:

$$
\{(y, g(y)): y \in \mathbb{R}\}=\left\{\left(v(k), 2 m_{0}^{2}(k) M(k)\right) / \pi: k \in[0,2 \pi)\right\}
$$

Here $m_{0}(k)$ is the density of the edge state at $k \in[0,2 \pi)$ defined by 4.2).

Now let's consider the following situation. Assume that we are given the distribution $\nu_{n}$ $(n>>1)$ in advance, which has been obtained by the measurement of the quantum state $\psi_{n}$; which is the $n$-th iteration of AE model with the initial state $\psi_{0}$. Here the parameters that determine the AE model; $r=\sin (\alpha-\beta)$ and $s=\sin (\alpha+\beta)$, are not known by us. In the rest of this section, we will try to estimate the group velocity $v(k)$ using only this $\nu_{n}$. First, in the following Corollary, we present a means of knowing $v(k)$ using the limit distribution of $\nu_{n} ; g(y)$. Secondly, we demonstrate a means of estimating $\nu_{n}$ using this Corollary (see Fig (7).

Corollary 4. Let $g(\cdot), r$ be the above and $C_{0}$ be $\int_{-\infty}^{\infty} g(y) d y$. Then the group velocity $v(k)$ satisfies the following:

$$
\begin{aligned}
& g(v(k)) \frac{d v(k)}{d k}=\frac{2 s^{2} / r^{2}}{\pi} v^{2}(k), \\
& \int_{0}^{2 \pi}|v(k)| \frac{d k}{2 \pi}=\frac{2 \arcsin |r|}{\pi}
\end{aligned}
$$

Here $s^{2}$ is expressed as

$$
s^{2}=\frac{r^{2}}{1-\sqrt{1-r^{2}}} C_{0} .
$$

The second equation is a kind of boundary condition of the above differential equation. The above Corollary 4 suggests that we can estimate the underlying spectral information of the quantum system from information obtained by observation.

Proof. Since the second moment of $f_{K}(x ; r)$ is $1-\sqrt{1-r^{2}}[23]$ and $f_{K}(x ;|r|)$ is an even function, then the total mass $C_{0}$ can be computed explicitly as (5.29). (5.27) comes directly from Corollary 3 and (5.18). Concerning the expression of $v(k)$ in (5.15), we divide the integration into four parts $\int_{0}^{\pi / 2}+\int_{\pi / 2}^{\pi}+\int_{\pi}^{3 \pi / 2}+\int_{3 \pi / 2}^{2 \pi}$ such that $v(k)$ is a monotonic function in each region. By (5.17),

$$
\int_{m \pi / 2}^{(m+1) \pi / 2}|v(k)| \frac{d k}{2 \pi}=\frac{1}{2} \int_{0}^{|r|} y f_{K}(y ; r) d y, \quad(m=0,1,2,3) .
$$

The RHS can be expressed as $\arcsin |r| /(2 \pi)$. Thus we have (5.28). 
Regarding the explicit expression for $g(x)$ in (5.3), we also notice from this linear differential equation for the group-velocity $v(k)=d \theta_{0} / d k$ that the group velocity of the edge state is expressed by an inverse of Konno's distribution $F_{K}(y ; r)=\int^{y} f_{K}(x ; r) d x$, that is,

$$
v(k)=F_{K}^{-1}\left(2 s^{2} k /\left(r^{2} \pi\right)+\zeta\right) .
$$

Here $\zeta$ satisfies

$$
\int_{0}^{2 \pi}\left|F_{K}^{-1}\left(2 s^{2} k /\left(r^{2} \pi\right)+\zeta\right)\right| \frac{d k}{2 \pi}=\frac{2 \arcsin |r|}{\pi} .
$$

Before closing this section, we compare numerical results for the probability of the selfloop at $[0, j]$ at the time step $n, \nu_{n}(j)$, with Theorem 3. In Figures 6 (a) and (b), we consider $0<|r|<1$ which gives continuously linear spreading behaviors. In Figures 6 (a-1) and (b1 ), we see that the scaled limit measure $g(y) / n$ in (5.3) runs through the "middle" of the oscillating $\nu_{n}(j)$. When we take the cumulation of $\nu_{n}(j)$ to $n y$, that is, $\sum_{j<n y} \nu_{n}(j)$, then in the right figures, we can see that the oscillation is almost wiped, and the cumulations of $\nu_{n}(j)$ and the integral of $g(y)$ almost overlap. This is a fundamental effect of the convergence in the distribution of Theorem 3 .

Next, we consider the ballistic spreading with $|r|=1$ and localization with $|r|=0$ in Figure 6 (c-1). In this case, Theorem 3 and the numerical results are almost consistent each other. We also confirm that the numerical result with $|r|=0$ at odd-time steps completely agrees with (5.7).

Finally, applying Corollary 4, we derive the group velocity $v(k)$ from the numerical result $\nu_{400}(j)$ in Figure 6 as an example. To this end, we need to extract $g(v(k)), r$, and $C_{0}$ from $\nu_{n}(j)$. The parameter $r$ is roughly estimated from Figure 6 and $C_{0}$ is calculated from $C_{0}=\sum_{j=0}^{400} \nu_{400}(j)$. To determine $g(v(k))$, we use the relation $y=j / n=v(k)$ and the fact that the cumulation of $\nu_{n}(y)$ shows fewer oscillations, making the numerical fittings stable. Assuming that the integral of $g(y)$ is well approximated by a polynomial whose lowest order is third, we have

$$
G(y)=\int_{0}^{y} g\left(y^{\prime}\right) d y^{\prime} \approx \begin{cases}\sum_{m=3}^{M} g_{m} y^{m} & : 0 \leq y \leq r \\ C_{0} & : y>r .\end{cases}
$$

We determine the coefficient $g_{m}$ up to the order $M=5$ by numerically fitting to the cumulation of $\nu_{n}(j)$ as shown in Figure $7($ a). We summarize the extracted values as follows:

$$
g_{3}=52.47 \pm 5.64, g_{4}=-483.16 \pm 54.24, g_{5}=1449.38 \pm 129.12, r=0.26, C_{0}=0.47456 .
$$

From (5.27) and (5.30), we obtain

$$
\sum_{m=3}^{M} \frac{m}{m-2} g_{m} y^{m-2}=\frac{2 s^{2} / r^{2}}{\pi}\left(k-k_{0}\right),
$$

where $k_{0}$ is a constant of integration. In the case of $M=5$, one of the general solutions of $y=v(k)$ is guaranteed to be real. Then, we determine $k_{0}$ such that it satisfies (5.28). In figure $7(\mathrm{~b})$, we compare the $|v(k)|$ obtained from the probability $n_{400}(j)$ with the exact solution in (5.15) and confirm that we can derive $|v(k)|$ from $\nu_{n}(j)$. 


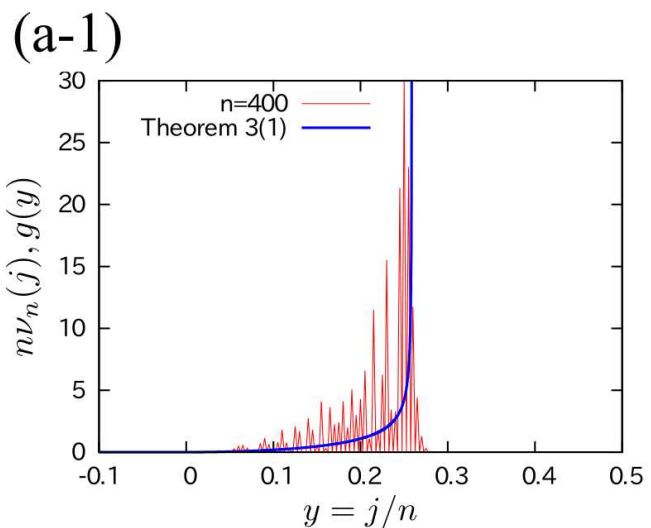

(b-1)

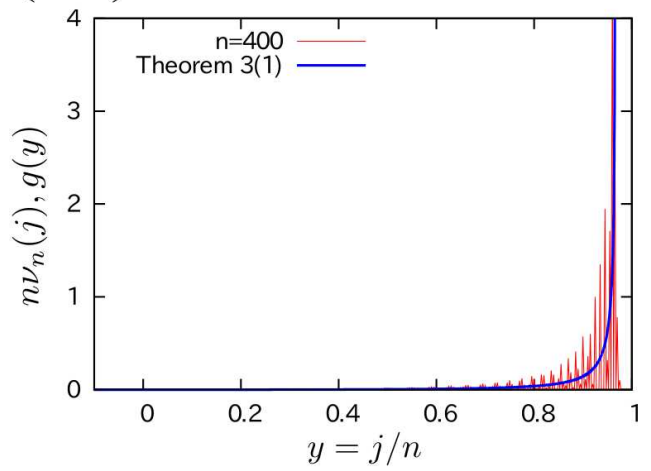

(c-1)

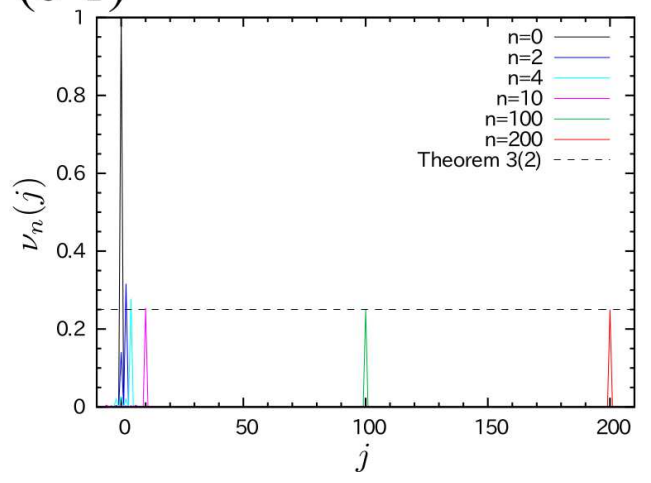

$(\mathrm{a}-2)$

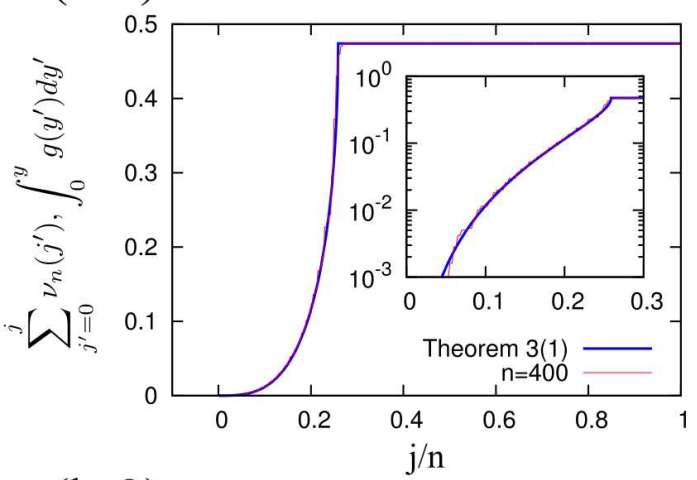

(b-2)

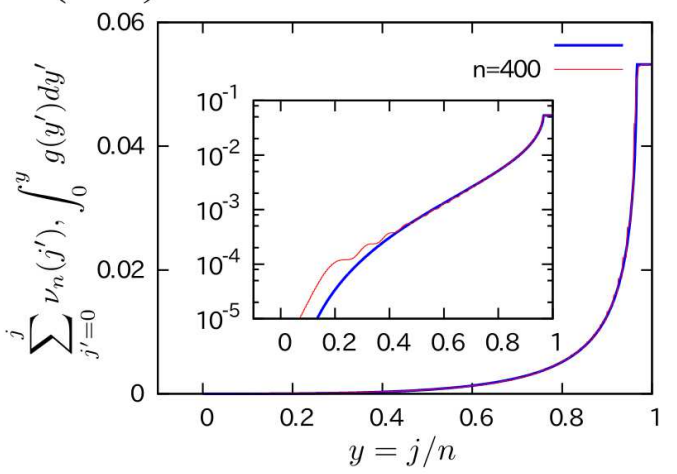

$(\mathrm{c}-2)$

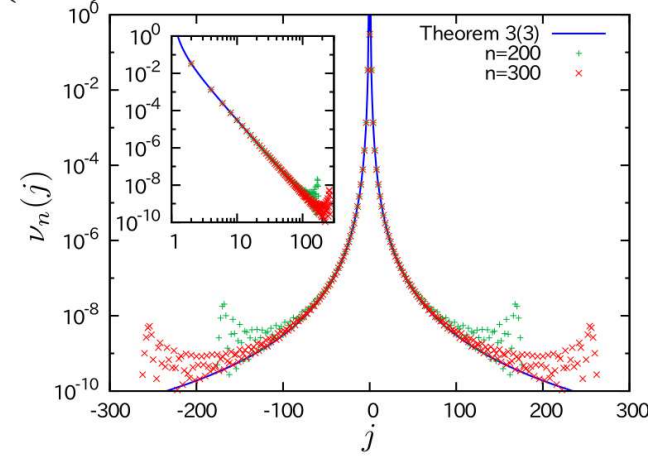

Figure 6: The rescaled measure on self-loops $n \nu_{n}(j)$ at $n=400$ time steps, as obtained by numerical simulations (a-1) and (b-1), and the cumulative distributions (thin red curves) are obtained by (a-2) and (b-2). Figures (a) and (b) are results for the case of $(\alpha, \beta)=(\pi / 4, \pi / 6)$ whose dispersion relations are given in (a-2) in Figure 4 and the case of $(\alpha, \beta)=(3 \pi / 4, \pi / 6)$, whose dispersion relations are given in (a-3) in Figure 4, respectively. The corresponding limit density, $g(y)$, in (5.3) and the distribution in (5.2) in Theorem 3 (1) are shown by thick blue curves. Inset of (a-2 and (b-2)): the semi-log plot of the distribution. (c): The measures on self-loops $n \nu_{n}(j)$ at various time steps, as obtained by the numerical simulations with (c-1) $(\alpha, \beta)=(5 \pi / 3, \pi / 6)$ and (c-2) $(\pi / 3, \pi / 3)$ with dispersion relations given in (a-2) and (b-2) in Figure 5, respectively. (c-1) The corresponding limit measures $\lim _{n \rightarrow \infty} \nu_{n}(j)$ in (5.5) in Theorem 3 (2) is shown by the dashed line. (c-2) The corresponding limit measures $\lim _{n \rightarrow \infty} \nu_{n}(j)$ in (5.6) in Theorem $3(3)$ is shown by the thick blue curves. Inset of (c-2): the log-log plot of the main figure. 

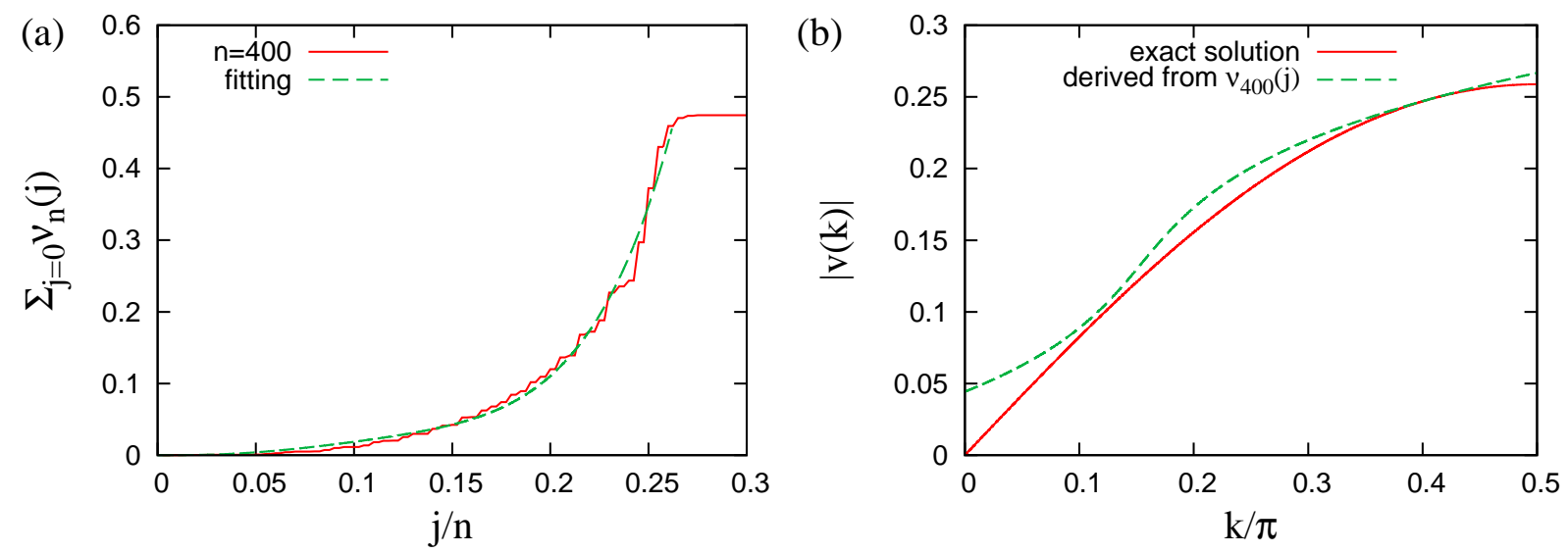

Figure 7: (a) The cumulation of $\nu_{400}(j)$ (solid red curve) for the $(\alpha, \beta)=(\pi / 4, \pi / 6$ ) parameter case and $G(y)=\sum_{m=3}^{5} g_{m} y^{m}$ obtained by the numerical fitting (dashed green curve). (b) The group velocity $|v(k)|$ derived from $\nu_{400}(j)$ with $k_{0}=-0.602$ (green dashed curve) and the exact solution in (5.15) (solid red curve).

\section{Limit distributions toward bulk}

In the previous section, we showed limit theorems for the finding probability at the self-loops on the boundary. In this section, we will show how the contribution of the edge states to the behavior of the quantum walk decays toward the bulk. To this end, we provide a limit theorem corresponding to Theorem 3 for the finding probability at other arcs for $0<|r|<1$ and $s \neq 0$ case.

We set $\varphi_{n}^{\prime}:=\hat{\Gamma}_{k}^{n} \varphi_{0}^{\prime}$ with $\varphi_{0}^{\prime}(j)=\delta(j)^{T}[0,1]$, where $\hat{\Gamma}: \ell^{2}\left(\mathbb{Z}_{+} ; \mathbb{C}^{2}\right) \rightarrow \ell^{2}\left(\mathbb{Z}_{+} ; \mathbb{C}^{2}\right)$ is defined in (3.2). Recall that we have shown that $\varphi_{n}^{\prime}$, which is the $n$-th iteration of the quantum walk on the Fourier space, is expressed by the $n$-th power of the CMV matrix $\mathcal{C}_{k}$ in (3.12), that is,

$$
\varphi_{n}^{\prime}=\Lambda_{k}^{-1}\left({ }^{T} \mathcal{C}_{k}\right)^{n} \Lambda_{k} \varphi_{0}^{\prime}
$$

We have

$$
\begin{aligned}
\varphi_{n}^{\prime}(j) & =\left[\begin{array}{c}
e^{-i w(2 j+1)}\left(\mathcal{C}_{k}\right)_{0,2 j+1} \\
e^{-i w(2 j)}\left(\mathcal{C}_{k}\right)_{0,2 j}
\end{array}\right] \\
& =\int_{|z|=1} z^{n}\left[\begin{array}{c}
e^{-i w(2 j+1)} x_{2 j+1}(z) \\
e^{-i w(2 j)} x_{2 j}(z)
\end{array}\right] d \mu(z),
\end{aligned}
$$

where $x_{j}(z)=\overline{\chi_{j}(1 / \bar{z})}$. The first equality derives from the definition of $\Lambda_{k}$ with $\left(\Lambda_{k} \varphi_{0}^{\prime}\right)(j)=$ $\delta(j)$ and $\left({ }^{T} \mathcal{C}_{k}\right)_{j, i}=\left(\mathcal{C}_{k}\right)_{i, j}$; the second equality is given by (5.9). This Lemma follows from [9, 25]:

Lemma 7. Let $\mathcal{C}$ be the $C M V$ matrix with the Verblunsky parameter $(\gamma, 0, \gamma, 0, \ldots)$. Assume $\operatorname{Re}(\gamma) \neq 0$ which is a necessary and sufficient condition for $\sigma_{p}(\mathcal{C}) \neq \emptyset$. Then $\sigma_{p}(\mathcal{C})=\left\{e^{i \beta}\right\}$ satisfies 4.11) and

$$
x_{2 j}\left(e^{i \beta}\right)=\lambda^{j}, x_{2 j+1}\left(e^{i \beta}\right)=\lambda^{2 j+1},
$$


where

$$
\lambda=\frac{\operatorname{sgn}(\operatorname{Re}(\gamma))}{\rho}\left(\sqrt{1-\operatorname{Im}^{2}(\gamma)}-|\operatorname{Re}(\gamma)|\right) .
$$

Lemma 7 and the Riemann-Lebesgue lemma imply

$$
\varphi_{n}^{\prime}(j)=e^{i n \theta_{0}(k)} m_{0}(k)\left[\begin{array}{c}
e^{-i w(2 j+1)} \lambda^{2 j+1}(k) \\
e^{-i w(2 j)} \lambda^{2 j}(k)
\end{array}\right]+o(1 / \sqrt{n}),
$$

where

$$
\lambda(k)=\frac{\operatorname{sgn}(\operatorname{Re}(\eta(k)))}{\rho(k)}\left(\sqrt{1-\operatorname{Im}^{2}(\eta(k))}-|\operatorname{Re}(\eta(k))|\right) .
$$

Recall that $U: \mathcal{A} \rightarrow \mathcal{A}$ is the unitary time evolution of the quantum walk and $\psi_{0} \in \mathcal{A}$ is the initial state. Also recall that the relabelling of each element of $A$ in Definition 1 . We set $\psi_{n}=U^{2 n} \psi_{0}$. For $(j, m) \in V$ and $d \in\{0,1\}$, the square modulus of $\psi_{n}((j, m) ; d)$ is the probability that we find the state $((j, m) ; d)$ after the $n$-th iteration of $U$ starting from $((0,0) ; 1)$. Thus we newly introduce the probability measure on $\nu_{n}: \mathbb{Z}_{+} \times \mathbb{Z} \rightarrow[0,1]$ such that

$$
\nu_{n}(2 j, m):=\left|\psi_{n}((j, m) ; 1)\right|^{2} \text { and } \nu_{n}(2 j+1, m):=\left|\psi_{n}((j, m) ; 0)\right|^{2} .
$$

Remark that $\nu_{n}(0, m)$ is identical to $\nu_{n}(m)$ as discussed in the previous section. We set the Fourier transform of them such that for $j \in \mathbb{Z}_{+}, n \in \mathbb{Z}_{+}$and $\xi \in \mathbb{R}$,

$$
\phi_{n}^{(j)}(\xi)=\sum_{m \in \mathbb{Z}} \nu_{n}(j, m) e^{i \xi m}
$$

Remark that $\phi_{n}(0, \xi)$ coincides with $\phi_{n}(\xi)$ in the previous section.

In the above discussion, we fixed $k$ and treated $\hat{\varphi}_{n}$ as $\varphi^{\prime}(\cdot)=\hat{\varphi}_{n}(\cdot ; k) \in \ell^{2}\left(\mathbb{Z}_{+} ; \mathbb{C}^{2}\right)$ whereas below, we will treat $\hat{\varphi}_{n}$ as a function of $k$ for fixed $j$, that is, $\hat{\varphi}_{n}(j ; \cdot) \in L^{2}\left([0,2 \pi) ; \mathbb{C}^{2}\right)$. We set $\hat{\varphi}_{n}(j ; k):={ }^{T}\left[\hat{\varphi}_{n, 0}(j ; k), \hat{\varphi}_{n, 1}(j ; k)\right]$, and obtain

$$
\begin{aligned}
\phi_{n}^{(2 j)}(\xi) & =\int_{0}^{2 \pi} \overline{\hat{\varphi}_{n, 1}(j ; k)} \hat{\varphi}_{n, 1}(j ; k+\xi) \frac{d k}{2 \pi}, \\
\phi_{n}^{(2 j+1)}(\xi) & =\int_{0}^{2 \pi} \overline{\hat{\varphi}_{n, 0}(j ; k)} \hat{\varphi}_{n, 0}(j ; k+\xi) \frac{d k}{2 \pi} .
\end{aligned}
$$

Since $r \neq 0$, by replacing $\xi$ with $\xi / n$ in the above equation and using expansion (6.2), we can obtain

$$
\left[\begin{array}{c}
\phi_{n}^{(2 j+1)}(\xi / n) \\
\phi_{n}^{(2 j)}(\xi / n)
\end{array}\right]=\int_{0}^{2 \pi} e^{i \xi v(k)} m_{0}^{2}(k)\left[\begin{array}{c}
\lambda^{2(j+1)}(k) \\
\lambda^{2 j}(k)
\end{array}\right] \frac{d k}{2 \pi}+o(1 / \sqrt{n}) .
$$

Since $0<|r|<1$, by putting $y=v(k)$ for $k \in[0,2 \pi)$, we have

$$
\lambda^{2}(k)=\frac{|r|-|s||y|}{|r|+|s||y|} .
$$

Combining the above with (5.17) and (5.18), we obtain

$$
\lim _{n \rightarrow \infty} \phi_{n}^{(j)}(\xi / n)=\int_{-\infty}^{\infty} e^{i \xi y} g(j, y) d y
$$


Here the measure $g: \mathbb{Z}_{+} \times \mathbb{R} \rightarrow \mathbb{R}_{+}$is denoted by

$$
\begin{array}{r}
g(2 j+1, y)=\frac{s^{2}}{r^{2}} y^{2} f_{K}(y ;|r|) \zeta^{j+1}(y) \times \begin{cases}\mathbf{1}_{[0, \infty)}(y) & : s r>0, \\
\mathbf{1}_{(-\infty, 0]}(y) & : s r<0,\end{cases} \\
g(2 j, y)=\frac{s^{2}}{r^{2}} y^{2} f_{K}(y ;|r|) \zeta^{j}(y) \times \begin{cases}\mathbf{1}_{[0, \infty)}(y) & : s r>0, \\
\mathbf{1}_{(-\infty, 0]}(y) & : s r<0,\end{cases}
\end{array}
$$

with

$$
\zeta(y)=\frac{|r|-|s||y|}{|r|+|s||y|}
$$

We summarize this section in the following theorem which extends the result of Theorem 3 (1) to the other $\operatorname{arcs}$ since $g(0, y)$ coincides with $g(y)$ given by (5.3):

Theorem 4. Let $\nu_{n}: \mathbb{Z}_{+} \times \mathbb{Z} \rightarrow[0,1]$ and $g: \mathbb{Z}_{+} \times \mathbb{R} \rightarrow \mathbb{R}_{+}$be the above. For $0<|r|<1$ with $s \neq 0$, we have

$$
\lim _{n \rightarrow \infty} \sum_{a<m / n<b} \nu_{n}(j, m)=\int_{a}^{b} g(j, y) d y \quad\left(j \in \mathbb{Z}_{+}\right)
$$

with $g(j, y)$ given by (6.6).

Remark that the limit measure $g(j, \cdot)$ has the support $[0,|r|)$ or $(-|r|, 0]$. If we insert $y=\operatorname{sgn}(r s) r$ into (6.7), then $\zeta(y)=(1-|s|) /(1+|s|)$ holds, which agrees with (B21) in [4]; this is the decay rate of the edge state wavefunctions towards the bulk for the zero quasi energy. The normalized position $y=\operatorname{sgn}(r s) r$ coincides with the maximal absolute value of the group-velocity $v(k)$. In such a wave number $k$, the effective mass $M(k)$, which is the inverse of the derivative of $v(k)$, diverges implying that the value $g(j ; y)$ also diverges, see Corollary 3 ,

We remark that the LHS in Theorem 4 for the $s=0$ case, which is equivalent to the condition lacking the edge state, becomes zero and also note that the limit measure $g$ : $\mathbb{Z}_{+} \times \mathbb{R} \rightarrow \mathbb{R}_{+}$is not a probability measure; we have focused on only the edge state's effect on the finding probability without considering on the bulk state. Indeed, putting $\Omega:=\mathbb{Z}_{+} \times \mathbb{R}$ and supposing $r s>0$, we have

$$
\begin{aligned}
\int_{\omega \in \Omega} g(\omega) d \omega & =\int_{-\infty}^{\infty} \sum_{j=0}^{\infty} g(j ; y) d y=\int_{0}^{|r|} \frac{1+\zeta(y)}{1-\zeta(y)} \frac{s^{2}}{r^{2}} y f_{K}(y ;|r|) d y \\
& =\int_{0}^{|r|} \frac{|s|}{|r|} y f_{K}(y ;|r|) d y \leq|s| / 2<1 .
\end{aligned}
$$

Consideration of the missing value of at least $1-|s| / 2$ which is the contribution of the bulk state may be an interesting subject for future work.

\section{Acknowledgments.}


NK were supported by the Grant-in-Aid for Scientific Research Challenging Exploratory Research (JSPS KAKENHI No. JP15K13443). ES acknowledges financial support from the Grant-in-Aid for Young Scientists (B) and of Scientific Research (B) Japan Society for the Promotion of Science (Grant No. 16K17637, No. 16K03939). HO was supported by a Grant-in-Aid for Scientific Research on Innovative Areas "Topological Materials Science" (JSPS KAKENHI No. JP16H00975) and also JSPS KAKENHI (No. JP16K17760 and No. JP16K05466). Finally, authors would like to thank reviewers for providing invaluable suggestions to this paper.

\section{References}

[1] Ambainis A, Bach E, Nayak A, Vishwanath A and Watrous J 2001 One-dimensional quantum walks Proc. 33rd Annual ACM Symp. Theory of Computing 37-49

[2] Asbóth J K 2012 Symmetries, topological phases, and bound states in the one-dimensional quantum walk Phys. Rev. B 86, 195414

[3] Asbóth J K and Obuse H 2013 Bulk-boundary correspondence for chiral symmetric quantum walks Phys. Rev. B 88, 121406(R)

[4] Asboth J K and Edge J M 2015 Edge-state-enhanced transport in a two-dimensional quantum walk Phys. Rev. A 91022324

[5] Avila J C, Schulz-Baldes H and Villegas-Blas C 2013, Topological invariants of edge states for periodic two-dimensional models, Math. Phys., Anal. Geom. 16 136-170

[6] Bernevig A B and Hughes T L 2013 Topological Insulators and Topological Supercnductors Princeton University Press, Princeton, NJ

[7] Cantero M J, Moral L and Velázquez L 2003 Five-diagonal matrices and zeros of orthogonal polynomials on the unit circle Linear Algebra and its Applications 362 29-56

[8] Cantero M J, Moral L and Velázquez L 2005 Minimal representations of unitary operators and orthogonal polynomials on the unit circle Linear Algebra and its Applications 405 40-65

[9] Cantero M J, Grünbaum F A, Moral L and Velázquez L 2010 Matrix valued Szegö polynomials and quantum random walks Communications on Pure and Applied Mathematics 63 464-507

[10] Cedzich C, Geib T, Grunbaum F A, Stahl C, Velazquez L, Werner A H, Werner R F 2016 The topological classification of one-dimensional symmetric quantum walks Preprint arXiv:1611.04439

[11] Cedzich C, Grunbaum F A, Stahl C, Velazquez L, Werner A H, Werner R F 2016 Bulk-edge correspondence of one-dimensional quantum walks Journal of Physics A: Mathematical and Theoretical 4921

[12] Chiu C K, Teo J C Y, Schnyder A P and Ryu S 2016 Classification of topological quantum matter with symmetries Rev. Mod. Phys. 88035005

[13] Endo T, Konno N, and Obuse H 2015 Relation between two-phase quantum walks and the topological invariant Preprint arXiv:1511.04230 
[14] Endo S, Endo T, Konno N, Segawa E and Takei M 2015 Limit theorems of a two-phase quantum walk with one defect Quantum Information and Computation 15 1373-1396

[15] Franco C Di, McGettrick M and Busch Th 2011 Mimicking the probability distribution of a two-dimensional Grover walk with a single-qubit coin Phys. Rev. Lett. 106080502

[16] Franco C Di, McGettrick M, Machida T and Busch Th 2011 Alternate two-dimensional quantum walk with a single-qubit coin Phys. Rev. A $\mathbf{8 4} 042337$

[17] Gudder S 1988 Quantum Probability (Academic Press Inc., CA)

[18] Graf G M and Porta M 2013 Bulk-edge correspondence for two-dimensional topological insulators, Commun. Math. Phys. 324 851-895.

[19] Hasan M Z and Kane C L 2010 Colloquium : Topological insulators Rev. Mod. Phys. 82 3045-3067

[20] Kitagawa T, Rudner M S, Berg E and Demler E 2010 Exploring topological phases with quantum walks Phys. Rev. A 82033429

[21] Kitagawa E, Berg E, Rudner M and Demler E 2010 Topological characterization of periodically driven quantum systems Phys. Rev. B 83235114

[22] Kitagawa T 2012 Topological phenomena in quantum walks; elementary introduction to the physics of topological phases Quantum Information Processing 11 1107-1148

[23] Konno N 2002 Quantum random walks in one dimension Quantum Inf. Proc. 1 345-354

[24] Konno N 2005 A new type of limit theorems for the one-dimensional quantum random walk J. Math. Soc. Jpn. 57 1179-1195

[25] Konno N and Segawa E 2011 Localization of discrete time quantum walks on a half line via the CGMV method Quantum Inf. Comput. 11 485-495

[26] Manouchehri K and Wang J B 2014 Physical Implementation of Quantum Walks (Quantum Science and Technology, Springer)

[27] Linder N H, Refael G and Galitski V 2011 Floquet topological insulator in semiconductor quantum wells Nature Physics 7490495

[28] Obuse H, Asbóth J K, Nishimura Y and Kawakami N 2015 Unveiling hidden topological phases of a one-dimensional Hadamard quantum walk Phys. Rev. B 92045424

[29] Obuse H and Kawakami N 2011 Topological phases and delocalization of quantum walks in random environments Phys. Rev. B 84195139

[30] Portugal R 2013 Quantum Walks and Search Algorithms (Quantum Science and Technology, Springer)

[31] Qi X Land Zhang S C 2011 Topological insulators and superconductors, Rev. Mod. Phys. 83 $1057-1110$

[32] Rudner M S, Lindner N H, Berg E, and Levin M 2013 Anomalous Edge States and the Bulk-Edge Correspondence for Periodically Driven Two-Dimensional Systems Phys. Rev. X 3 031005 
[33] Schnyder A P, Ryu S, Furusaki A, and Ludwig A W W 2008 Classification of topological insulators and superconductors in three spatial dimensions Phys. Rev. B 78195125

[34] Schulz-Baldes H and Villegas-Blas C 2016 Krein signatures of transfer operators for half-space topological insulators, J. Phys. A: Math. Theor. 49405201.

[35] Schnyder A P and Ryu S 2011 Topological phases and surface flat bands in superconductors without inversion symmetry Phys. Rev. B $84060504(\mathrm{R})$

[36] Volovik G E 2007 Quantum phase transitions from topology in momentum space Lect. Notes Phys. 718 31-73

\section{Appendix A Proof of Lemma 1}

We set $C^{\prime}=\mathcal{U} C \mathcal{U}^{-1}$ and $S^{\prime}=\mathcal{U} S \mathcal{U}^{-1}$. By the bipartiteness of the coin operator $C$ with respect to the " $\{0,1\}$ " and " $\{2,3\}$ " directions, it holds that for every $\phi \in \mathcal{A}$,

$$
\left[\begin{array}{l}
(C \phi)(\boldsymbol{x} ; 0) \\
(C \phi)(\boldsymbol{x} ; 1)
\end{array}\right]=\sigma_{1} H_{\alpha}\left[\begin{array}{l}
\phi(\boldsymbol{x} ; 2) \\
\phi(\boldsymbol{x} ; 3)
\end{array}\right],\left[\begin{array}{l}
(C \phi)(\boldsymbol{x} ; 2) \\
(C \phi)(\boldsymbol{x} ; 3)
\end{array}\right]=\sigma_{1} H_{\beta}\left[\begin{array}{c}
\phi(\boldsymbol{x} ; 0) \\
\phi(\boldsymbol{x} ; 1)
\end{array}\right] .
$$

Here $\sigma_{1}=|0\rangle\langle 1|+| 1\rangle\langle 0|$. Using this, for every $\psi \in \ell^{2}\left(V ; \mathbb{C}^{4}\right)$ with $\psi(\boldsymbol{x})={ }^{T}\left[\psi_{0}(\boldsymbol{x}), \ldots, \psi_{3}(\boldsymbol{x})\right]$, we have

$$
\left(C^{\prime} \psi\right)(\boldsymbol{x})=|0\rangle \otimes \sigma_{1} H_{\alpha}\left[\begin{array}{l}
\psi_{2}(\boldsymbol{x}) \\
\psi_{3}(\boldsymbol{x})
\end{array}\right]+|1\rangle \otimes \sigma_{1} H_{\beta}\left[\begin{array}{l}
\psi_{0}(\boldsymbol{x}) \\
\psi_{1}(\boldsymbol{x})
\end{array}\right] .
$$

Concerning the shift operator $S$ flipping the arc direction; $(S \phi)(a)=\phi(\bar{a})$, we have

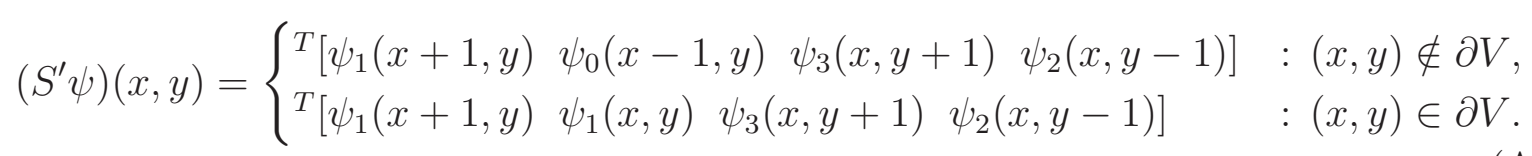

By (A.2) and (A.3), we have

$$
\begin{aligned}
\left(U^{\prime} \psi\right)(x, y)=\tilde{P}_{\alpha} \psi^{(\uparrow)}(x+1, y) & +\tilde{Q}_{\alpha} \psi^{(\uparrow)}(x-1, y) \\
& +\tilde{P}_{\beta} \psi^{(\leftrightarrow)}(x, y+1)+\tilde{Q}_{\beta} \psi^{(\leftrightarrow)}(x, y-1), \quad((x, y) \notin \partial V),
\end{aligned}
$$

and

$$
\begin{aligned}
\left(U^{\prime} \psi\right)(x, y)=\tilde{P}_{\alpha} \psi^{(\uparrow)}(x+1, y) & +\tilde{S}_{\alpha} \psi^{(\uparrow)}(x, y) \\
& +\tilde{P}_{\beta} \psi^{(\leftrightarrow)}(x, y+1)+\tilde{Q}_{\beta} \psi^{(\leftrightarrow)}(x, y-1), \quad((x, y) \in \partial V) .
\end{aligned}
$$

Then we prove the lemma.

\section{Appendix B Proof of Lemma 2}

For $\psi=\psi^{(\leftrightarrow)} \in \ell^{2}\left(V ; \mathbb{C}^{4}\right)$, we first examine $\left(U^{\prime 2} \psi\right)(x, y)$. We should remark that

$$
\left(U^{\prime} \psi^{(\leftrightarrow)}\right)(x, y)=\left(U^{\prime} \psi\right)^{(\uparrow)}(x, y),
$$


because of $\tilde{Q}_{\beta}$ and $\tilde{P}_{\beta}$. Thus by (2.4) in Lemma 1, for $(x, y) \notin \partial V$, we have

$$
\left(U^{\prime 2} \psi\right)(x, y)=\tilde{P}_{\alpha}\left(U^{\prime} \psi\right)^{(\uparrow)}(x+1, y)+\tilde{Q}_{\alpha}\left(U^{\prime} \psi\right)^{(\uparrow)}(x-1, y) .
$$

Moreover by (2.3) in Lemma 1 and (A.1), we have

$$
\begin{aligned}
\left(U^{\prime 2} \psi\right)(x, y)=\tilde{P}_{\alpha}\left(\tilde{Q}_{\beta} \psi^{(\leftrightarrow)}(x+\right. & \left.1, y-1)+\tilde{P}_{\beta} \psi^{(\leftrightarrow)}(x+1, y+1)\right) \\
& +\tilde{Q}_{\alpha}\left(\tilde{Q}_{\beta} \psi^{(\leftrightarrow)}(x-1, y-1)+\tilde{P}_{\beta} \psi^{(\leftrightarrow)}(x-1, y+1)\right) .
\end{aligned}
$$

Since $\psi$ is expressed by some $\varphi \in \mathcal{U}_{e}\left(\mathcal{A}^{(\leftrightarrow)}\right)$ such that $\psi(\boldsymbol{x})=|0\rangle \otimes \varphi(\boldsymbol{x})$ and it holds $\tilde{X}_{\alpha} \tilde{Y}_{\beta}=|0\rangle\langle 0| \otimes X_{\alpha} Y_{\beta}(X, Y \in\{P, Q\})$, we have

$$
\begin{aligned}
\left(U^{\prime 2} \psi\right)(x, y)=|0\rangle \otimes\left\{P_{\alpha} Q_{\beta} \varphi(x+\right. & \left.1, y-1)+P_{\alpha} P_{\beta} \varphi(x+1, y+1)\right) \\
& \left.\left.+Q_{\alpha} Q_{\beta} \varphi(x-1, y-1)+Q_{\alpha} P_{\beta} \varphi(x-1, y+1)\right)\right\} .
\end{aligned}
$$

In the same way for the $(x, y) \in \partial V$ case,

$$
\begin{aligned}
\left(U^{\prime 2} \psi\right)(x, y)=|0\rangle \otimes\left\{P_{\alpha} Q_{\beta} \varphi(x+1, y-1)\right. & \left.+P_{\alpha} P_{\beta} \varphi(x+1, y-1)\right) \\
& \left.\left.+S_{\alpha} Q_{\beta} \varphi(x, y-1)+S_{\alpha} P_{\beta} \varphi(x, y+1)\right)\right\}
\end{aligned}
$$

which implies that

$$
\left(U^{\prime 2} \psi\right)(\boldsymbol{x})=|0\rangle \otimes(\Gamma \varphi)(\boldsymbol{x}), \quad(\forall \boldsymbol{x} \in V) .
$$

By the way, for any $\phi \in \mathcal{A}^{(\leftrightarrow)}$, we have

$$
\left(\left.U^{2}\right|_{\mathcal{A}^{(\leftrightarrow)}} \phi\right)(\boldsymbol{x} ; j)=\left(\mathcal{U}^{-1}{U^{\prime}}^{2} \mathcal{U} \phi\right)(\boldsymbol{x}, j)= \begin{cases}\left(U^{\prime 2} \psi\right)_{j}(\boldsymbol{x})=(\Gamma \varphi)_{j}(\boldsymbol{x}) & : j \in\{0,1\} \\ 0 & : j \in\{2,3\} .\end{cases}
$$

where $\psi(\boldsymbol{x})=(\mathcal{U} \phi)(\boldsymbol{x})=|0\rangle \otimes \varphi(\boldsymbol{x})$. Here in the last equality, we used (A.6) $)$. Remarking $\mathcal{U U}_{e}^{-1} \varphi=\varphi$, we have

$$
\left(\left.\mathcal{U}_{e} U^{2}\right|_{\mathcal{A}^{(\leftrightarrow)}} \mathcal{U}_{e}^{-1} \varphi\right)(\boldsymbol{x})=\left[\begin{array}{l}
\left(\left.U^{2}\right|_{\mathcal{A}^{(\leftrightarrow)}} \mathcal{U}_{e}^{-1} \varphi\right)(\boldsymbol{x} ; 0) \\
\left(\left.U^{2}\right|_{\mathcal{A}^{(\leftrightarrow)}} \mathcal{U}_{e}^{-1} \varphi\right)(\boldsymbol{x} ; 1)
\end{array}\right]=\left[\begin{array}{l}
(\Gamma \varphi)_{0}(\boldsymbol{x}) \\
(\Gamma \varphi)_{1}(\boldsymbol{x})
\end{array}\right]=(\Gamma \varphi)(\boldsymbol{x}),
$$

which completes the proof.

\section{Appendix C Connection between the moving shift and flip-flop shift representations of the quan- tum walk}

For clarity, we mention the connection between the above description of the coin and the shift operators $C^{\prime}$ and $S^{\prime}$ in (A.2) and (A.3), which is so called the flip-flop shift representation, and the commonly used description of the coin and the shift operators, $C^{\prime \prime}$ and $S^{\prime \prime}$, respectively such as in Ref. [4], which is so called the moving shift representaion. We regard four internal states in the vertex representation as the left, right, down, and up moving components,

$$
|L\rangle={ }^{T}[1,0,0,0], \quad|R\rangle={ }^{T}[0,1,0,0], \quad|D\rangle={ }^{T}[0,0,1,0], \quad|U\rangle={ }^{T}[0,0,0,1],
$$


respectively, and the position $(x, y)$ is described by

$$
|x, y\rangle \text {. }
$$

On this basis, the coin operator $C^{\prime}$ is described by

$$
C^{\prime}=\sum_{x, y}|x, y\rangle\langle x, y| \otimes\left[\begin{array}{cc}
0 & \sigma_{1} H_{\alpha} \\
\sigma_{1} H_{\beta} & 0
\end{array}\right] \quad:(x, y) \in V .
$$

while the common descriptions of $C^{\prime \prime}$ and $S^{\prime \prime}$ are

$$
C^{\prime \prime}=\sum_{x, y}|x, y\rangle\langle x, y| \otimes\left[\begin{array}{cc}
0 & H_{\alpha} \\
H_{\beta} & 0
\end{array}\right] \quad:(x, y) \in V .
$$

and

$$
\begin{aligned}
& S^{\prime \prime}|x, y\rangle|R\rangle=|x+1, y\rangle|R\rangle, \\
& S^{\prime \prime}|x, y\rangle|D\rangle=|x, y-1\rangle|D\rangle, \\
& S^{\prime \prime}|x, y\rangle|U\rangle=|x, y+1\rangle|U\rangle, \\
& S^{\prime \prime}|x, y\rangle|L\rangle= \begin{cases}|x-1, y\rangle|L\rangle & :(x, y) \notin \partial V, \\
|x, y\rangle|R\rangle & :(x, y) \in \partial V,\end{cases}
\end{aligned}
$$

respectively. Rewriting the time-evolution operator $U^{\prime}$, we have

$$
U^{\prime}=S^{\prime} C^{\prime}=S^{\prime \prime}\left(S^{\prime \prime-1} S^{\prime} C^{\prime}\right)
$$

Taking into account the different order of internal states in the basis, $S^{\prime \prime-1} S^{\prime}$ is expressed as

$$
S^{\prime \prime-1} S^{\prime}=\sum_{x, y}|x, y\rangle\langle x, y| \otimes\left[\begin{array}{cc}
\sigma_{1} & 0 \\
0 & \sigma_{1}
\end{array}\right]
$$

and we confirm the relation

$$
C^{\prime \prime}=S^{\prime \prime-1} S^{\prime} C^{\prime}
$$

From (A.2) and (A.3), without loss of generality, we can alternatively describe the model as $U^{\prime}=S^{\prime \prime} C^{\prime \prime}$ instead of $U^{\prime}=S^{\prime} C^{\prime}$ in the vertex representation. 